\title{
Tetracycline-Inactivating Enzymes
}

\author{
Jana L. Markley and Timothy A. Wencewicz* \\ Department of Chemistry, Washington University in St. Louis, St. Louis, MO, United States
}

Tetracyclines have been foundational antibacterial agents for more than 70 years. Renewed interest in tetracycline antibiotics is being driven by advancements in tetracycline synthesis and strategic scaffold modifications designed to overcome established clinical resistance mechanisms including efflux and ribosome protection. Emerging new resistance mechanisms, including enzymatic antibiotic inactivation, threaten recent progress on bringing these next-generation tetracyclines to the clinic. Here we review the current state of knowledge on the structure, mechanism, and inhibition of tetracycline-inactivating enzymes.

Keywords: tetracycline destructases, enzymatic antibiotic inactivation, antibiotic adjuvants, tetracyclines, antibiotic resistance, flavin monooxygenase

\section{OPEN ACCESS INTRODUCTION}

\section{Edited by: \\ Graeme L. Conn, \\ Emory University School of Medicine, \\ United States \\ Reviewed by: \\ Pablo Sobrado, \\ Virginia Tech, United States \\ Yury S. Polikanov, \\ The University of Illinois at Chicago, \\ United States \\ *Correspondence: \\ Timothy A. Wencewicz \\ wencewicz@wustl.edu \\ Specialty section: \\ This article was submitted to \\ Antimicrobials, Resistance \\ and Chemotherapy, \\ a section of the journal \\ Frontiers in Microbiology \\ Received: 01 March 2018 \\ Accepted: 04 May 2018 \\ Published: 30 May 2018 \\ Citation: \\ Markley $J L$ and Wencewicz TA \\ (2018) Tetracycline-Inactivating \\ Enzymes. Front. Microbiol. 9:1058. \\ doi: 10.3389/fmicb.2018.01058}

\section{Tetracycline Antibiotics}

Tetracycline antibiotics were discovered in the 1940s and found widespread clinical use shortly thereafter (Duggar, 1948; Finlay et al., 1950; King et al., 1950; Roberts, 1996; Nelson and Levy, 2011). Naturally occurring tetracyclines are highly oxidized, type II polyketides composed of a linear fused tetracyclic scaffold with rings designated A, B, C, D (Figure 1; Stephens et al., 1952; Chopra and Roberts, 2001). Tetracyclines inhibit bacterial protein synthesis by binding to the $16 \mathrm{~S}$ rRNA of the $30 \mathrm{~S}$ bacterial ribosome subunit, preventing accommodation of incoming aminoacyltRNAs at the acceptor site (A-site) (Brodersen et al., 2000; Wilson, 2009). Tetracyclines make sequence-independent contacts with sugar phosphates in the primary binding site between h31 and h34. Both synthetic and semisynthetic tetracyclines have found clinical use as low cost, broadspectrum, and orally available antimicrobial agents. The minimum active pharmacophore for bacterial ribosome inhibition is 6-deoxy-6-demethyltetracycline (McCormick et al., 1960; Chopra and Roberts, 2001). Chemical modification of positions 5-9 is well tolerated and can improve ribosome affinity, as is the case for the first and second generation scaffolds CTc and doxycycline. Modification of positions 1-4 and 10-12 strongly attenuates the antibacterial activity. The 1,3diketone group at carbons 11 and $12(\mathrm{pKa} \sim 7)$ chelates $\mathrm{Mg}^{2+}$ (Stephens et al., 1956; Jin et al., 2007). The tetracycline-Mg complex is the biologically active form that permeates the bacterial cell envelope (Schnappinger and Hillen, 1996) and binds to bacterial ribosomes (Jenner et al., 2013), transcription factors (Hinrichs et al., 1994), and aptamers (Xiao et al., 2008).

Chemical modification of the tetracycline scaffold has preserved this important class of antibiotics for $>70$ years against continuous waves of resistance determinants (Charest et al., 2005; Liu and Myers, 2016; Sun and Xiao, 2017). The unique 3D chemical shape of tetracycline arises

Abbreviations: ARG, antibiotic resistance gene; CTc, chlortetracycline; FAD, flavin adenine dinucleotide; FMO, flavin monooxygenase; NADPH, nicotinamide adenine dinucleotide phosphate reduced form; $\mathrm{NADP}^{+}$, nicotinamide adenine dinucleotide phosphate oxidized form. 
from a bend in the structure at the A,B-ring juncture, and this seems to be a distinguishing feature from other tetracyclic polyketides that impart selectivity for ribosome binding (Brodersen et al., 2000; Stepanek et al., 2016). The D-ring of tetracyclines has proven to be robust toward semi-synthetic modifications, as highlighted by the bulky $N$ - $t$-butyl-glycylamide side chain of the third generation antibiotic tigecycline, which plays a dual role in overcoming resistance and increasing affinity for the 30S ribosomal subunit (Jenner et al., 2013). Access to fully synthetic tetracyclines, including fourth generation compounds eravacycline (Ronn et al., 2013; Zhanel et al., 2016) and omadacycline (Tanaka et al., 2016) - both currently in phase III clinical trials - has rejuvenated clinical prospects for this drug class (Liu and Myers, 2016; Sun and Xiao, 2017). With the approval of next-generation tetracyclines on the horizon, new mechanisms of tetracycline resistance are certain to emerge as clinical use increases. Our ability to manage emerging resistance is critical to ensure future utility of tetracycline antibiotics and prevent a public health care crisis (Brown and Wright, 2016).

\section{Tetracycline Resistance}

Resistance to tetracycline antibiotics was observed from the very start of clinical use (Schiott and Stenderup, 1954; Roberts, 1996). Despite widespread clinical resistance, tetracyclines continue to be important agents for treating a variety of human infections caused by Gram-negative and Gram-positive bacterial pathogens, along with atypical pathogens including mycoplasmas, nematodes, and parasitic protozoans (Chopra and Roberts, 2001). Tetracyclines are also widely used in veterinary medicine and agricultural applications, including crop protection and intensive animal farming, which has contributed to the widespread dissemination of tetracycline resistance (Thaker et al., 2010; Surette and Wright, 2017). Molecular mechanisms of tetracycline resistance include efflux (Izaki and Arima, 1963; Levy and McMurry, 1978; Kaneko et al., 1985), ribosome protection proteins (Burdett et al., 1982; Burdett, 1986, 1991, 1996), reduced permeability (Cohen et al., 1988), ribosome mutation (Ross et al., 1998), and enzymatic inactivation (Figure 2; Yang et al., 2004; Nguyen et al., 2014). Efflux pumps and ribosome protection proteins are the most common types of clinical resistance to tetracyclines and have been found in most human pathogens (Connell et al., 2003; Piddock, 2006; Thaker et al., 2010). Seven groups of efflux pumps have been identified that confer tetracycline resistance by decreasing the effective intracellular antibiotic concentration, with most members falling into the major facilitator superfamily (Guillaume et al., 2004; Piddock, 2006). Ribosome protection proteins are GTPases with homology to elongation factors that bind the ribosome analogously to elongation factors and chase bound tetracycline from the $30 \mathrm{~S}$ ribosomal subunit (Connell et al., 2003; Jenner et al., 2013). Reduced drug permeability is achieved through morphological changes and the modification or reduced expression of porins and likely contributes to clinical tetracycline resistance (Cohen et al., 1988; Schnappinger and Hillen, 1996; Olesky et al., 2006; Justice et al., 2008). Ribosomal mutations are uncommon in clinical resistance to tetracyclines, probably due to the sequence-independent binding mode of tetracycline
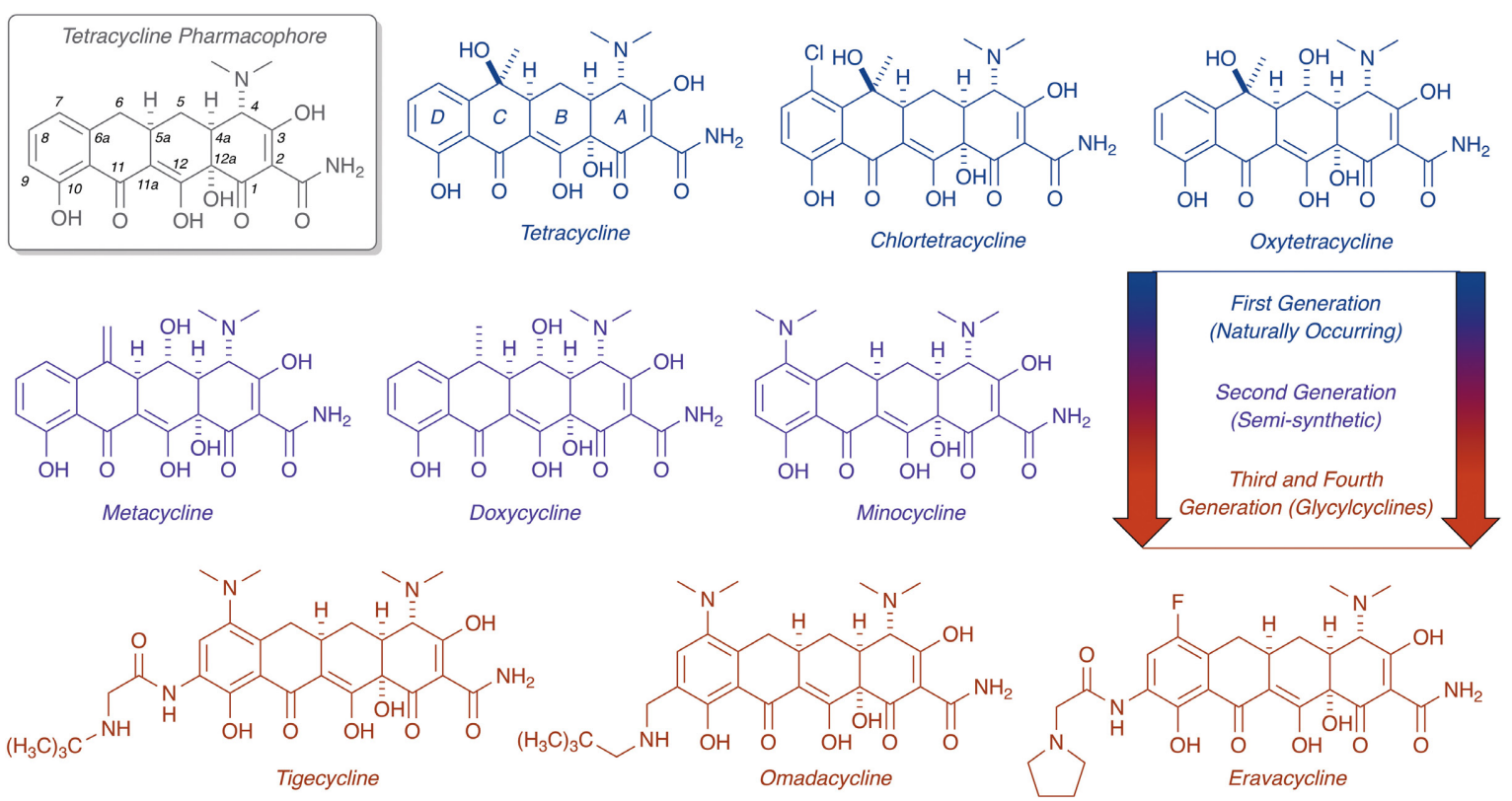

FIGURE 1 | Evolution of the tetracycline scaffold. 6-Deoxy-6-demethyltetracycline represents the minimum tetracycline pharmacophore required for inhibition of the ribosome. Tetracycline (first reported in 1953), CTc (first reported in 1948), and oxytetracycline (first reported in 1950) represent first generation structures. Metacycline (first reported in 1962), doxycycline (first reported in 1967), and minocycline (first reported in 1961) represent second generation structures. Tigecycline (first reported in 1993) is the only FDA-approved third generation structure, while omadacycline (first reported in 2013) and eravacycline (first reported in 2013) are fourth generation molecules currently in phase III clinical trials. 


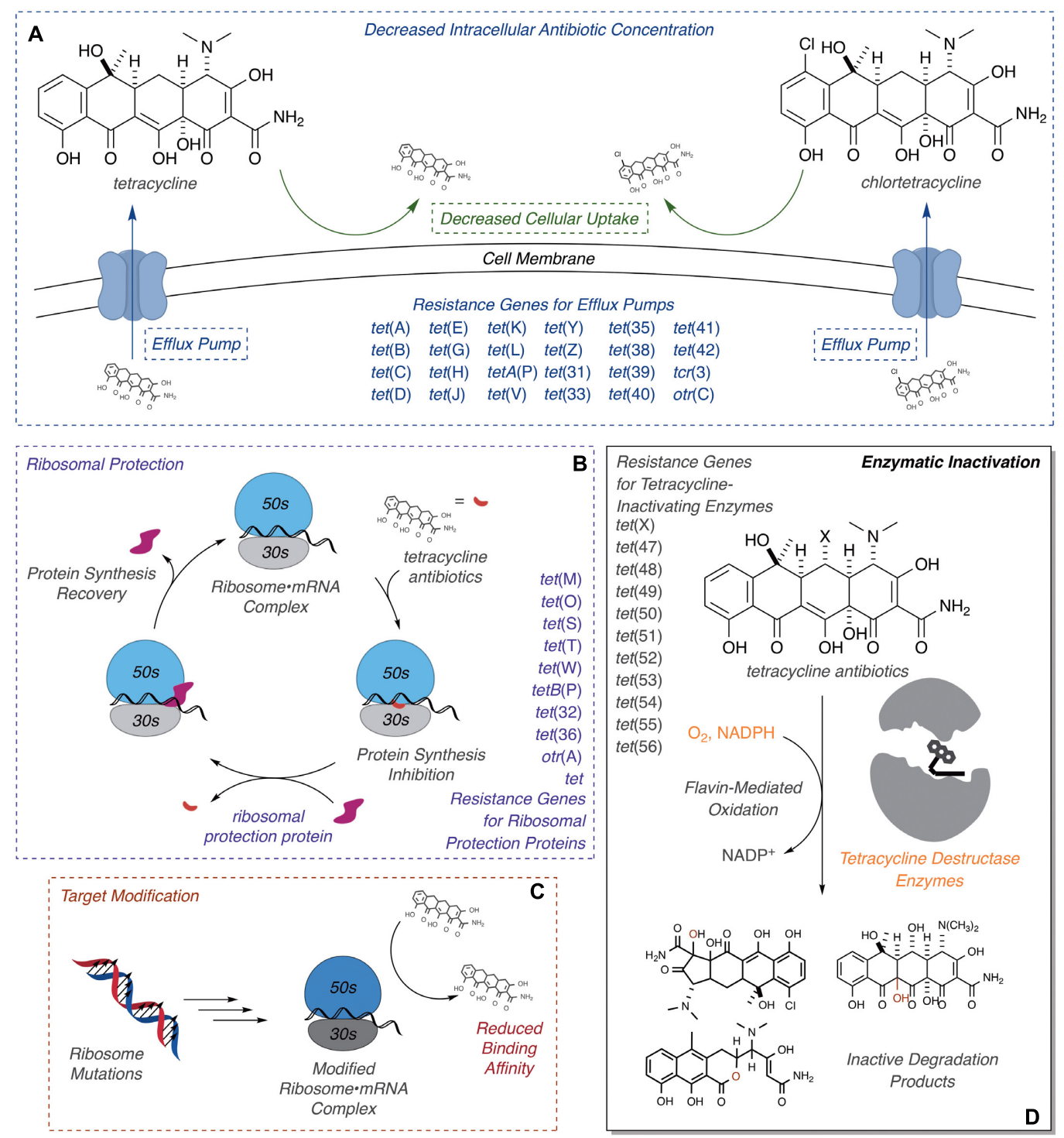

FIGURE 2 | Molecular mechanisms of tetracycline resistance. (A) Efflux, exclusion, (B) ribosome protection, (C) ribosome modification, and (D) enzymatic inactivation. Documented ARGs associated with each type of tetracycline resistance are provided.

to the 30 S ribosomal subunit (Brodersen et al., 2000); still, some resistance-conferring mutations and deletions around the tetracycline-binding site have been reported (Ross et al., 1998; Gerritis et al., 2002; Trieber and Taylor, 2002). Some clinical isolates of Helicobacter pylori (Nonaka et al., 2005) and Propionibacterium acnes (Ross et al., 1998) carry point mutations in the $16 \mathrm{~S}$ ribosome that confer tetracycline resistance, presumably through reduced tetracycline binding affinity. These ribosome mutations also confer tetracycline resistance in laboratory strains of Escherichia coli (Cocozaki et al., 2016). Similar resistance to tigecycline in S. pneumonia, arising from point mutations in ribosomal proteins and rRNA, has been introduced in the laboratory via successive passaging (Lupien et al., 2015). A more obscure resistance mechanism involves activation of $\mathrm{Mg}^{2+}$-dependent purine nucleotide biosynthesis via expression of the tet34 gene product, a predicted xanthineguanine phosphoribosyltransferase, which attenuates tetracycline antibacterial activity presumably by increasing the pool of GTP available to elongation factors to accelerate binding of aminoacyltRNAs to the 30S ribosomal subunit (Nonaka and Suzuki, 2002; Kim et al., 2003).

Third (tigecycline) and fourth generation (eravacycline and omadacycline) tetracyclines are known to overcome resistance via efflux and ribosome protection (Jenner et al., 2013; Zhanel et al., 2016; Tanaka et al., 2016). However, enzymatic inactivation has emerged as a new concern for these next-generation tetracyclines (Moore et al., 2005; Grossman et al., 2012, 2017). A family of FMOs, the tetracycline destructases (Forsberg et al., 2015), has been shown to selectively oxidize tetracyclines leading to covalent destruction of the antibiotic scaffold 
(Yang et al., 2004). Unlike efflux, exclusion, ribosome protection, and ribosome modification, enzymatic inactivation permanently eliminates the tetracycline antibiotic challenge by decreasing intracellular and extracellular antibiotic concentrations (Davies, 1994; Wright, 2005). The clinical impact of enzymatic antibiotic inactivation can be devastating, as documented by the spread of broad-spectrum beta-lactamases across the globe (Bush and Jacoby, 2010; Brandt et al., 2017). The goal of this review is to highlight recent advances involving the structure, mechanism, and inhibition of tetracycline destructases to bring awareness and inspire solutions for this emerging type of tetracycline resistance.

\section{TETRACYCLINE DESTRUCTASES}

\section{Antibiotic Destructases}

The tetracycline destructases are part of a broadly defined family of enzymes, which we are calling the antibiotic destructases, that inactivate antibiotics via a wide variety of covalent modifications to the antibiotic scaffold (Davies, 1994; Wright, 2005). Antibiotic destructases are named to reflect the enzymatic activity associated with covalent modification of antibiotic scaffolds that permanently destroys antimicrobial activity and imparts resistance to producing microbes. Antibiotic destructases differ from xenobiotic modifying metabolic enzymes in regulation, catalytic efficiency, rate, and substrate specificity. Xenobiotic modifying enzymes perform housekeeping functions in the host, primarily clearance, and detoxification of xenobiotics (Krueger and Williams, 2005). The primary function of antibiotic destructases is gain of resistance. Thus, xenobiotic modifying enzymes tend to be broad in substrate scope at the cost of catalytic efficiency, while antibiotic destructases tend to be narrower in substrate scope with high specificity and catalytic efficiency toward a particular structural class of antibiotics (Wright, 2005).

Well-known examples of antibiotic destructases include beta-lactamases that hydrolyze the strained 4-membered lactam of beta-lactam antibiotics (Bush and Jacoby, 2010; Brandt et al., 2017), and aminoglycoside-inactivating enzymes including phosphotransferases, acetyltransferases, and adenylyltransferases that modify the free amine and hydroxyl groups of aminoglycoside antibiotics (Ramirez and Tolmasky, 2010). Known classes of antibiotic destructases (antibiotic substrates) include peptidases (bogorol, bacitracin) (Li et al., 2018), hydrolases (beta-lactams, macrolides) (Bush and Jacoby, 2010; Morar et al., 2012), thioltransferases (fosfomycin) (Rife et al., 2002; Thompson et al., 2013), epoxidases (fosfomycin) (Fillgrove et al., 2003), cyclopropanases (colibactin) (Tripathi et al., 2017), acyl transferases (aminoglycosides, chloramphenicol, glufosinate, tabtoxinine-beta-lactam, streptogramin) (Leslie, 1990; Botterman et al., 1991; Sugantino and Roderick, 2002; Ramirez and Tolmasky, 2010; Wencewicz and Walsh, 2012; Favrot et al., 2016), methyl transferases (holomycin) (Li et al., 2012; Warrier et al., 2016), nucleotidylyl transferases (aminoglycosides, lincosamide) (Morar et al., 2009; Ramirez and Tolmasky, 2010), ADP-ribosyltransferases (rifamycins) (Baysarowich et al., 2008), glycosyltransferases (aminoglycosides, rifamycins, macrolides) (Bolam et al., 2007; Ramirez and Tolmasky,
2010; Spanogiannopoulos et al., 2012), phosphotransferases (aminoglycosides, chloramphenicol, rifamycins, macrolides, viomycin) (Thiara and Cundliffe, 1995; Izard and Ellis, 2000; Ramirez and Tolmasky, 2010; Stogios et al., 2016; Fong et al., 2017), lyases (streptogramins) (Korczynska et al., 2007), and oxidoreductases (tetracyclines, rifamycins) (Park et al., 2017; Koteva et al., 2018). As antibiotic prospecting continues, the list of antibiotic destructases is certain to grow (Crofts et al., 2017; Li et al., 2018; Pawlowski et al., 2018).

Unlike other major classes of antibiotic resistance (efflux, exclusion, target modification), covalent inactivation by antibiotic destructases permanently neutralizes the antibiotic challenge and lowers intracellular and extracellular antibiotic concentrations. If antibiotic levels fall below the MIC, then resistance is achieved. Covalent modification of antibiotics can perturb target affinity, block cellular uptake, trigger efflux mechanisms, or lead to decomposition of the antibiotic (Wright, 2005). Genes encoding for antibiotic destructases are often present in operons that are co-transcribed with biosynthetic genes in the antibiotic producing microbe (Li et al., 2018). Co-transcription ensures self-protection during antibiotic biosynthesis (Bolam et al., 2007; Mack et al., 2014). Antibiotic destructases are often transferable through mobilized genetic elements such as plasmids (Davies and Davies, 2010). Once transformed into a host microbial cell, the expression of antibiotic destructases is often inducible and in some cases can be triggered specifically in response to antibiotic challenge (Llarrull et al., 2011). Antibiotic destructases can be excreted to the periplasm or even the extracellular space in order to destroy the antibiotic before it reaches the microbial cell. Resistance caused by antibiotic destructases can be overcome, in theory, by modifying the antibiotic scaffold to evade destructases (Syriopoulou et al., 1981), co-administration of a destructase inhibitor (Drawz and Bonomo, 2010), inhibition of destructase production or localization (Therien et al., 2012), or increasing intracellular concentrations to overcome destructase production (McPherson et al., 2012). Thus far, only modification of the antibiotic scaffold and co-administration of a destructase inhibitor have proven effective for overcoming resistance by antibiotic destructases in clinical infections (Fisher et al., 2005; Drawz and Bonomo, 2010).

Each class of antibiotic destructase represents a distinct chemical mode of antibiotic inactivation with the evolutionary potential to broaden or narrow substrate discrimination (Pawlowski et al., 2018). The evolutionary landscape leans heavily in favor of optimizing resistance enzymes due to the widespread selective pressure applied by broad-spectrum antibiotics. To prepare and respond to the emergence of antibiotic destructases, a thorough understanding of the genetic origins, dissemination, structure, and mechanism of the antibiotic destructase must be established. The rise of beta-lactamases in hospital- and community-acquired infections is the historical model for resistance via antibiotic destruction. Continuous innovation around the beta-lactam pharmacophore and co-administration of beta-lactamase inhibitors as adjuvants has maintained the clinical viability of this important antibiotic class (Bush, 2018). Most of the antibiotic-inactivating enzymes cited above do not represent 
current clinical threats; however, each threatens to emerge pending widespread clinical use of the corresponding antibiotic class. The recent success of fourth generation tetracyclines in advanced clinical trials has raised concerns over selecting for tetracycline destructases that might compromise future clinical use of the entire tetracycline class of antibiotics.

\section{TetX - The Flagship}

\section{Tetracycline-Inactivating Enzyme}

Enzymatic inactivation of tetracyclines was first proposed as a resistance mechanism in 1984 (Guiney et al., 1984). A plasmid that conferred tetracycline resistance to $E$. coli with a strict requirement for aerobic growth was isolated from the human commensal Bacteroides fragilis (Matthews and Guiney, 1986; Park et al., 1987; Speer and Salyers, 1988). Plasmid mapping revealed the presence of a putative tetracycline efflux pump and a gene, tet $X$, encoding for a potentially novel tetracycline resistance enzyme that catalyzes tetracycline degradation (Park and Levy, 1988). Growth of E. coli carrying the tet $X$ gene on an inducible plasmid yielded a distinctive brown colored growth phenotype, exclusively under aerobic conditions (Speer and Salyers, 1989). Spent media from tetracycline-treated cultures of $E$. coli expressing the tet $X$ gene showed decreased tetracycline concentrations and loss of tetracycline activity against wild-type E. coli. Cell-free lysates of $E$. coli expressing tet $X$ strictly required exogenous NADPH for tetracycline inactivation, consistent with TetX being an NADPH-dependent oxidoreductase (Speer et al., 1991). Two additional variants of the tet $X$ gene, tet $X 1$ and tet $X 2$, were later identified in another Bacteroides transposon (Whittle et al., 2001).

In 2004, Wright and coworkers heterologously expressed TetX, TetX1, and TetX2 in E. coli and purified the recombinant proteins (Yang et al., 2004). TetX and TetX2 are 99\% sequence identical, and both proteins co-purified with a bound flavin cofactor and proved to be active FMOs that degrade tetracyclines. TetX1 is a truncated variant that does not bind flavin and is thus not a true tetracycline resistance enzyme. TetX was shown to inactivate first, second, and third generation tetracyclines including tigecycline (Moore et al., 2005). Oxidation of oxytetracycline by TetX leads to formation of a variety of degradation products, including hydroxylation at $\mathrm{C} 11 \mathrm{a}$, the product of which was isolated and characterized by Wright and coworkers following an acid quench that provided the stabilized cyclic hemiketal (Figure 3A; alternate sites of oxidation and mechanistic considerations are discussed below in the section "Mechanisms of Tetracycline Oxidation") (Yang et al., 2004). Presumably, modification at C11a will attenuate $\mathrm{Mg}^{2+}$ chelation and ribosome binding, which are both required for biological activity of oxytetracycline (Schnappinger and Hillen, 1996). Additionally, hydroxylation of C11a destabilizes
A<smiles>CN(C)[C@H]1C(O)=C(C(N)=O)C(=O)[C@@]2(O)C(O)=C3C(=O)c4c(O)cccc4[C@@](C)(O)[C@H]3[C@@H](O)[C@H]12</smiles>

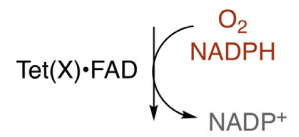

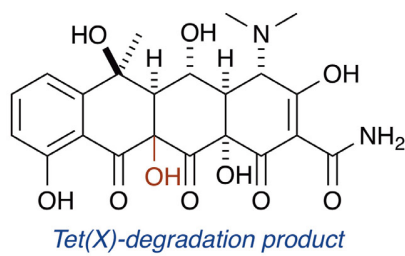

$\underset{\text { quench }}{\text { acid }} \downarrow p H=1$

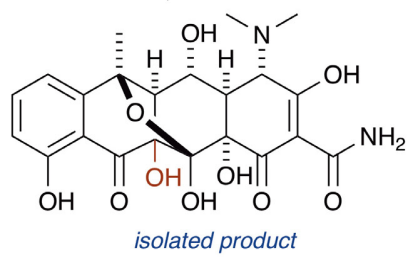

B

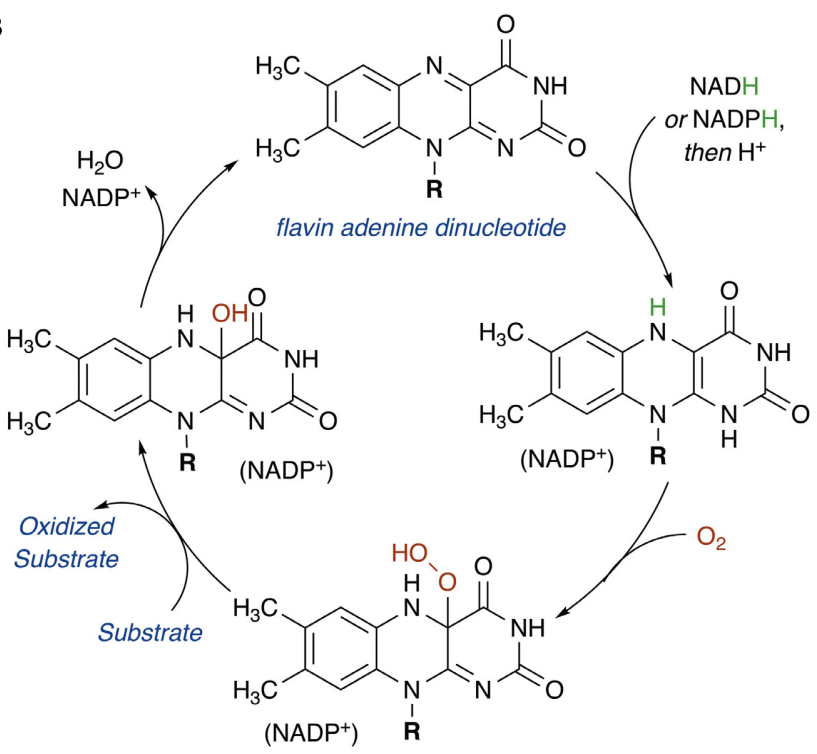

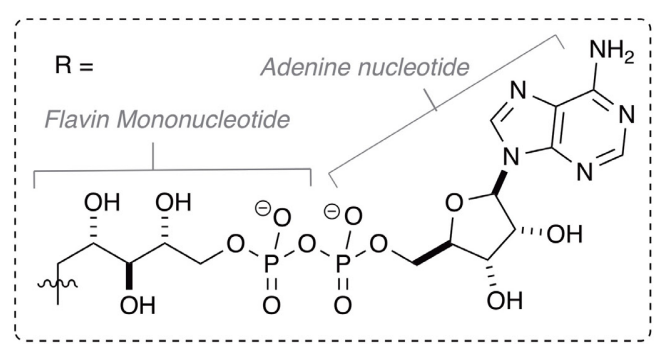

FIGURE 3 | (A) Hydroxylation of oxytetracycline by TetX. (B) Mechanism of class A FMOs. 
the tetracycline scaffold leading to complex mixtures of nonenzymatic degradation products (Yang et al., 2004). X-ray crystal structures of TetX bound to 7-CTc, 7-iodotetracycline, minocycline, and tigecycline have been reported and confirmed TetX to be a class A FMO (structures are discussed below in the section "Structural Basis for Tetracycline Inactivation") (Volkers et al., 2011, 2013). Similar to other class A FMOs, TetX is predicted to utilize NADPH to reduce the flavin cofactor in preparation for subsequent formation of a reactive $\mathrm{C} 4 \mathrm{a}$ peroxyflavin that transfers an electrophilic hydroxyl group to the nucleophilic C11a of the tetracycline enol (Figure 3B; van Berkel et al., 2006).

\section{Expanding the Tetracycline Destructase Family}

Mobilization of tet $X$ on transposons in Bacteroides suggests that dissemination of the tetracycline resistance gene into human pathogens is possible (Whittle et al., 2002). Indeed, in 2013, the tet $X$ gene was found in a variety of MDR Gram-negative pathogens (Enterobacter cloacae, Comamonas testosteroni, E. coli, Klebsiella pneumonia, Delftia acidovorans, and other members of Enterobacteriaceae and Pseudomonadaceae) isolated from a hospital in Sierra Leone (Leski et al., 2013). Several of the tetX harboring pathogens are on the CDC's list of ESKAPE pathogens (Santajit and Indrawattana, 2016), including Pseudomonas aeruginosa and Acinetobacter baumannii (Aminov, 2009, 2013; Deng et al., 2014). Although tetX has been found in human pathogens, there is yet to be a documented clinical case of tetracycline resistance caused by tet $X$ or related genes encoding for tetracycline destructases. The tet $X$ gene has also been observed in a variety of environmental bacteria, including Myroides odoratimimus (Ming et al., 2017), Sphingobacterium sp. (Ghosh et al., 2009, 2014), and Flavobacterium psychrophilum (Duchaud et al., 2018), and metagenomic samples including Chinese soil (Wang et al., 2017), human feces (Ohashi and Fujisawa, 2017), and hospital wastewater (Wang et al., 2018). The tet $X$ gene is encountered in a wide range of ecosystems (human gut, soil, hospital wastewater) and is present on mobile genetic elements primed for horizontal gene transfer. This pattern of ARG dissemination is consistent with horizontal gene transfer of tet $X$ between environmental bacteria and human pathogens, as has been observed for many other classes of ARGs (D'Costa et al., 2006; Forsberg et al., 2012; Crofts et al., 2017).

In 2015, a comprehensive functional metagenomic survey using tetracycline selection identified a new family of tet $X$ homologs denoted as the tetracycline destructases (tet49-tet55) (Forsberg et al., 2015). The novel tetracycline destructase genes showed at most $24.4 \%$ amino acid sequence homology to TetX. Cloning, heterologous expression, and in vitro characterization of Tet49-Tet55 revealed that all nine enzymes were functional tetracycline-inactivating FMOs. Comparative gene analysis revealed a tenth tetracycline destructase gene, tet56, in the genome of the human pathogen Legionella longbeachae. Antibacterial susceptibility and in vitro tetracycline degradation assays proved that tet56 is a true ARG that confers tetracycline resistance when expressed in L. longbeachae.
This expanded set of tetracycline destructases provided a unique opportunity to systematically explore substrate selectivity, characterize degradation products, screen for inhibitors, and compare structural features across the enzyme family. These studies led to several important crystal structures of Tet50, Tet51, Tet55, and Tet56 in a variety of functional states (see section "Structural Basis for Tetracycline Inactivation") that provide mechanistic insight on the diverse oxidation patterns at play for tetracycline substrates (the section "Mechanisms of Tetracycline Oxidation"). These studies also led to the discovery of the first pan tetracycline destructase inhibitor that rescues tetracycline activity when co-administered to tetracycline destructase expressing bacteria (see section "Tetracycline Destructase Inhibitors, an Adjuvant Approach”) (Park et al., 2017).

\section{STRUCTURAL BASIS FOR TETRACYCLINE INACTIVATION}

\section{Anatomy of a Tetracycline Destructase}

TetX and all members of the tetracycline destructase family are structural homologs of class A FMOs. Class A FMOs are single component flavoprotein hydroxylases that utilize FAD cofactors and $\mathrm{NAD}(\mathrm{P}) \mathrm{H}$ electron donors to oxidize small molecule substrates-primarily through electrophilic hydroxylation of electron-rich olefins or aromatic rings by a transient, catalytic C4a-hydroperoxyflavin (vide supra, Figure 3) (van Berkel et al., 2006; Montersino and Berkel, 2013; Huijbers et al., 2014; Mascotti et al., 2016; Romero et al., 2018). In general, this particular type of FMO enzyme is characterized by a single Rossmann fold that binds FAD through non-covalent interactions with the adenosine monophosphate moiety, which is linked to the catalytic isoalloxazine fragment via a polyoxygenated alkyl chain. Flexibility in this alkyl linker is fundamentally important to the success of the catalytic cycle, which involves multiple, dynamic conformational changes in enzyme structure to establish distinct functional enzyme states differentiated by relative FAD activation and three-dimensional orientation (vide infra). TetX and members of the tetracycline destructase family are structurally similar and functionally homologous (Forsberg et al., 2015). As shown in Figure 4, the tetracycline-inactivating FMO enzymes are composed of two major domains - a lower FAD-binding domain (green) that exhibits the characteristic Rossmann fold and an upper tetracycline binding domain (pink) (Volkers et al., 2011; Park et al., 2017). The association of the two domains is stabilized by a C-terminal alphahelix (purple), and specifically in the case of the tetracycline destructase family, a second C-terminal alpha-helix (cyan) is present near the tetracycline binding site, which plays an important role in substrate recognition and loading (Park et al., 2017).

While the exact sequence of events involving dynamic conformational changes to the enzyme during the catalytic cycle are currently unknown (see section "Tetracycline Destructase Inhibitors, an Adjuvant Approach" for a proposed model), two enzyme conformers have been observed via X-ray 

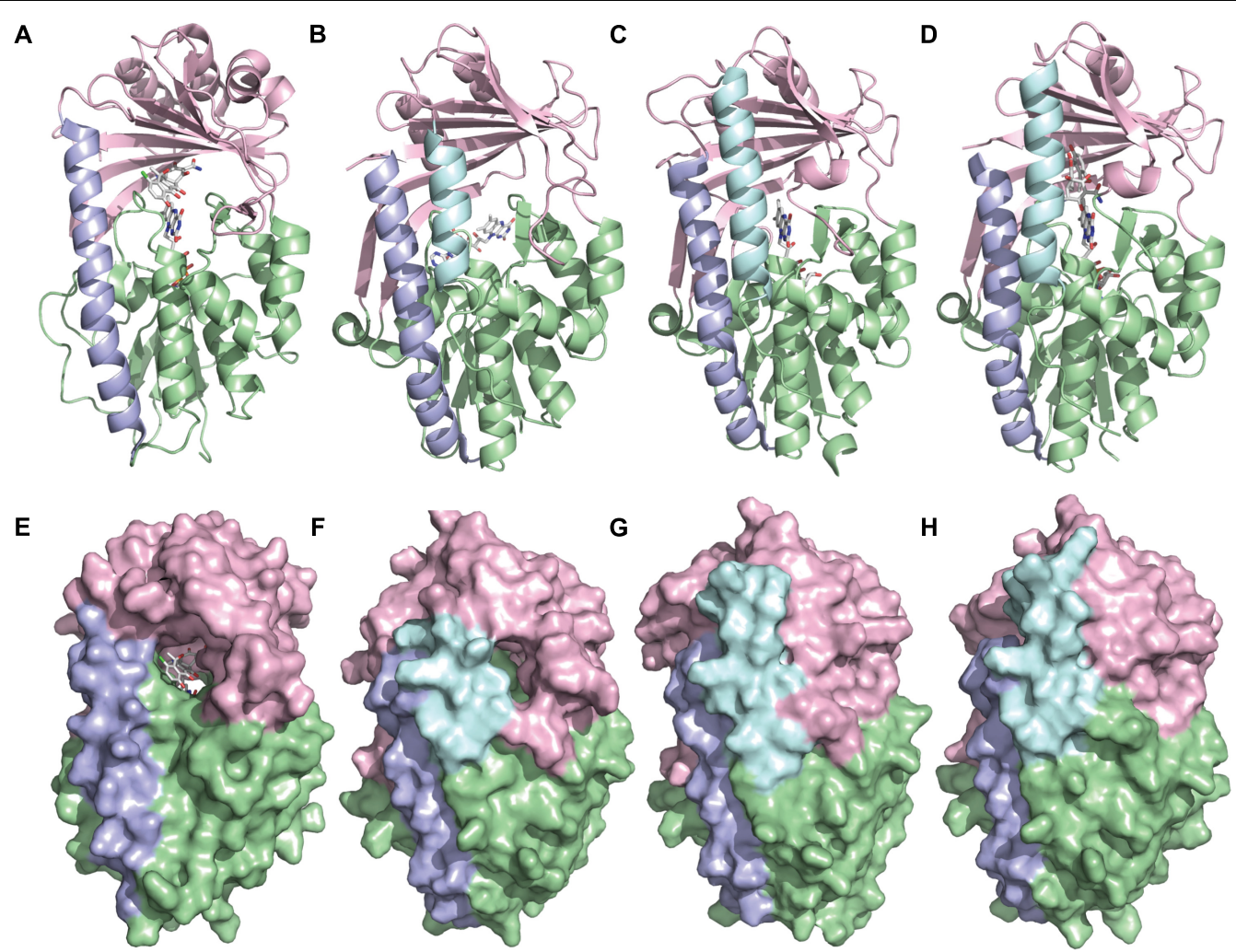

FIGURE 4 |X-ray crystal structure of a tetracycline destructase with bound tetracycline substrate and flavin cofactor. The mobility of the flavin cofactor is highlighted by showing the FAD-IN and FAD-OUT conformations observed during structural studies. (A) X-ray crystal structure of CTc bound to TetX (FAD-IN conformation, PDB ID: 2y6r). (B) X-ray crystal structure of Tet50 with no bound substrate (FAD-OUT conformation, PDB ID: 5tue). (C) X-ray crystal structure of Tet50 with no bound substrate (FAD-IN conformation, PDB ID: 5tue). (D) X-ray crystal structure of CTc bound to Tet50 (FAD-IN conformation, PDB ID: 5 tui). (E) Surface view of X-ray crystal structure of CTc bound to TetX (FAD-IN conformation, PDB ID: 2y6r). (F) Surface view of X-ray crystal structure of Tet50 with no bound substrate (FAD-OUT conformation, PDB ID: 5tue). (G) Surface view of X-ray crystal structure of Tet50 with no bound substrate (FAD-IN conformation, PDB ID: 5tue). (H) Surface view of X-ray crystal structure of CTc bound to Tet50 (FAD-IN conformation, PDB ID: 5tui). Images were generated using PyMOL v1.7.

crystallographic analysis which are distinct in both FADorientation and tertiary protein structure (Figure 4). The FAD-OUT conformer, in which the substrate loading channel is open and the FAD cofactor is pointed away from the tetracycline binding domain, allows for easy accommodation of the substrate and ready access of FAD to electron-donor $\mathrm{NADPH}$ to maintain a steady concentration of reduced $\mathrm{FADH}_{2}$ primed for reactivation with molecular oxygen (shown for Tet50, Figure 4B, surface view Figure 4F). While the FADOUT conformation has not been experimentally observed for TetX, it has been observed in other class A-type FMOenzymes (particularly StaC and RebC) (Ryan et al., 2008; Goldman et al., 2012) and is fundamentally important to maintain catalytic efficiency and relevant levels of antibiotic resistance.

Upon substrate and/or NADPH accommodation, several class A FMO enzymes undergo a series of discrete conformational changes that flip the activated $\mathrm{FADH}_{2}$ toward the bound substrate and allow for both the protected formation of the reactive $\mathrm{C} 4 \mathrm{a}$-peroxyflavin from $\mathrm{FADH}_{2}$ and molecular oxygen and subsequent substrate oxidation (Ghisla and Massey, 1989; Palfey and McDonald, 2010; Montersino and Berkel, 2013;
Huijbers et al., 2014). However, this FAD-IN conformer has been observed via X-ray crystallography for TetX and Tet50 in the absence of NADPH and substrate. A defined sequence of mechanistic events has been elucidated for prototypical class A FMO p-hydroxybenzoate hydroxylase (Eppink et al., 1998, 1999; Suemori, 2013). While the tetracycline-inactivating enzymes appear to be class A FMOs, the defined sequence of events, including NADPH-binding elements, and relevant extrapolation of these no-substrate, FAD-IN conformers to solution-phase enzyme dynamic processes remain currently unknown. Nevertheless, X-ray crystallographic analysis of the no substrate- and substrate-bound FAD-IN conformers of Tet50 and the substrate-bound FAD-IN conformer of TetX highlights several structural differences that may aid in the explanation of the unique, enzyme-specific antibiotic resistance phenotypes observed in vitro and in whole cell for each of these tetracyclineinactivating enzymes (Forsberg et al., 2015; Park et al., 2017).

In the absence of the second C-terminal "gatekeeper" helix observed in members of the tetracycline destructase families, the FAD-IN conformation for CTc bound to TetX utilizes several hydrophobic, mostly aromatic residues to shield the FAD-complex from C4a-peroxyflavin-reactive solvent molecules 
(Figure 4A; Volkers et al., 2011). Indeed, the FAD cofactor is barely visible in the surface view of the CTc-bound, FADIN conformer of TetX (Figure 4E). However, a small, open pocket near the substrate-binding site allows for a portion of the substrate - in this case, CTc - to extend from the active site of the enzyme into solvent exposed space. In contrast, the substrate loading channel closes completely in the no substrateand CTc-bound FAD-IN conformers of Tet50, where both the FAD and the substrate are shielded from solvent interaction by both the "gatekeeper helix" and a hydrophobic phenylalanine residue in the substrate-binding domain (Phe95, Figures 4C,D) (Park et al., 2017). This structural difference between FAD-IN conformers of TetX and Tet50 is highlighted in the surface views of each protein conformer shown in Figure 4 (TetX Figure 4E and Tet50 Figures 4G,H). In addition, the structure variability in FAD-IN conformation has important implications in substrate recognition and binding, as well as enzymesubstrate specificity and preference, that directly result in distinct tetracycline degradation profiles.

\section{Diverse Substrate-Binding Modes}

As is the case with most class A FMO enzymes (van Berkel et al., 2006; Montersino and Berkel, 2013; Huijbers et al., 2014; Romero et al., 2018), the position of substrate oxidation is heavily dependent on the spatial orientation of the bound substrate in relation to the transient enzyme-associated C4aperoxyflavin cofactor. Because active site flexibility can lead to product mixtures (as multiple binding modes can lead to multiple degradation products), it is important to correlate experimentally observed binding modes with potential sites of substrate oxidation that correspond to characterized oxidation products.

In this regard, the X-ray crystal structure of CTc bound to TetX can serve as a point of reference to help define spatial coordinates within the enzyme active site in which the tetracycline substrate can rotate/bind (Figure 5; Volkers et al., 2011). As is shown in Figure $\mathbf{5 A}$, enzyme-bound $\mathrm{CTc}$ is located above the FAD cofactor, which is extended toward the substrate-binding domain within the enzyme active site, as is consistent with the FADIN conformation. In addition, CTc is oriented in such a way that the A-ring ( $\mathrm{C} 1$ proximal, $\mathrm{C} 4$ distal) is closest to the FAD cofactor, while the $\mathrm{D}$-ring lies nearer the $\mathrm{C}$-terminal alpha-helix (C10 proximal, C7 distal). Thus, this orientation can be defined as mode $\mathrm{I}_{\mathrm{D}, \mathrm{A}}$, where the mode number (I or II) describes the proximal or distal position of the $\mathrm{C} 1-\mathrm{C} 10$ hemisphere of the molecule in relation to the FAD cofactor, and the subscript identifier describes the west-to-east (left-to-right) association of the $\mathrm{D}$ - and A-rings of the tetracycline substrate in relation to the FAD cofactor. Correspondingly, a $180^{\circ}$ horizontal rotation of the tetracycline substrate bound in mode $\mathrm{I}_{\mathrm{D}, \mathrm{A}}$ (about a vertical axis) would result in substrate-binding mode $\mathrm{I}_{\mathrm{A}, \mathrm{D}}$ (C1,D-ring proximal), where the sole modification in binding mode is the relation of the A-ring (now west) and D-ring (now east) to the FAD cofactor (Figure 5B). The association of the $\mathrm{C} 1-\mathrm{C} 10$ hemisphere to the FAD isoalloxazine remains unchanged. In contrast, a $180^{\circ}$ vertical rotation (about a horizontal axis) of the tetracycline substrate bound in mode $\mathrm{I}_{\mathrm{D}, \mathrm{A}}$ would result in the substrate-binding mode $\mathrm{II}_{\mathrm{D}, \mathrm{A}}$, where the sole modification in binding mode is the relation of the $\mathrm{C} 1-\mathrm{C} 10$ hemisphere of the molecule in relation to the FAD cofactor (now distal). The association of the A- and D-rings to the FAD isoalloxazine remains unchanged. In this way, four potential binding modes for tetracycline substrates - modes $\mathrm{I}_{\mathrm{D}, \mathrm{A}}, \mathrm{I}_{\mathrm{A}, \mathrm{D}}, \mathrm{II}_{\mathrm{D}, \mathrm{A}}$, and $\mathrm{II}_{\mathrm{A}, \mathrm{D}}$ can be defined, and the oxidation of the tetracycline substrate will be binding-mode specific.

Of the four potential substrate-binding modes, only two have been experimentally observed via X-ray crystallography for the binding of substrates to tetracycline-inactivating enzymes (Figure 6; Volkers et al., 2011; Park et al., 2017). As described in the previous paragraph, CTc binds to TetX in mode $\mathrm{I}_{\mathrm{D}, \mathrm{A}}$, and the primary substrate recognition elements are located in the substrate-binding domain, where hydrogen-bond donating residues (Q192, H234, and $\mathrm{R} 213$ ) interact with $\mathrm{H}$-bond accepting ketone and amide functional groups on the A-ring of CTc
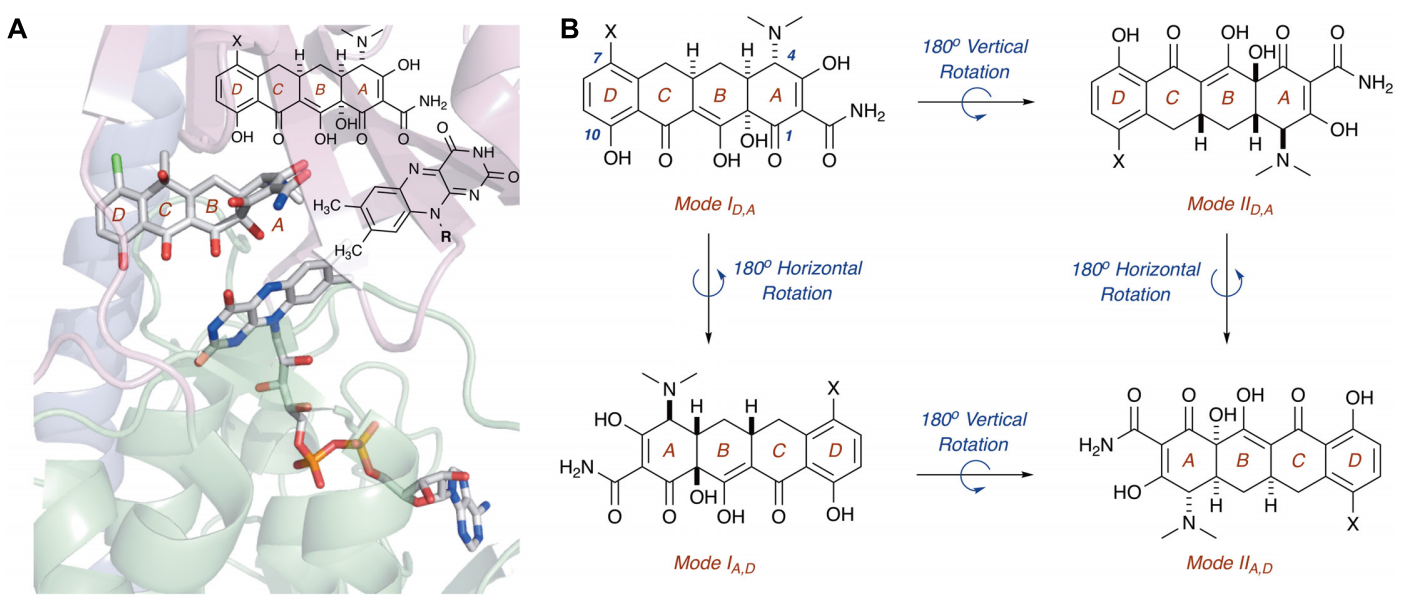

FIGURE 5 | (A) X-ray crystal structure of CTc bound to TetX in binding mode ID,A defines the orientation of FAD relative to each CTc binding mode (PDB ID: 2y6r). (B) Theoretical and experimentally observed tetracycline binding modes (four total). Image in panel (A) was generated using PyMOL v1.7. 


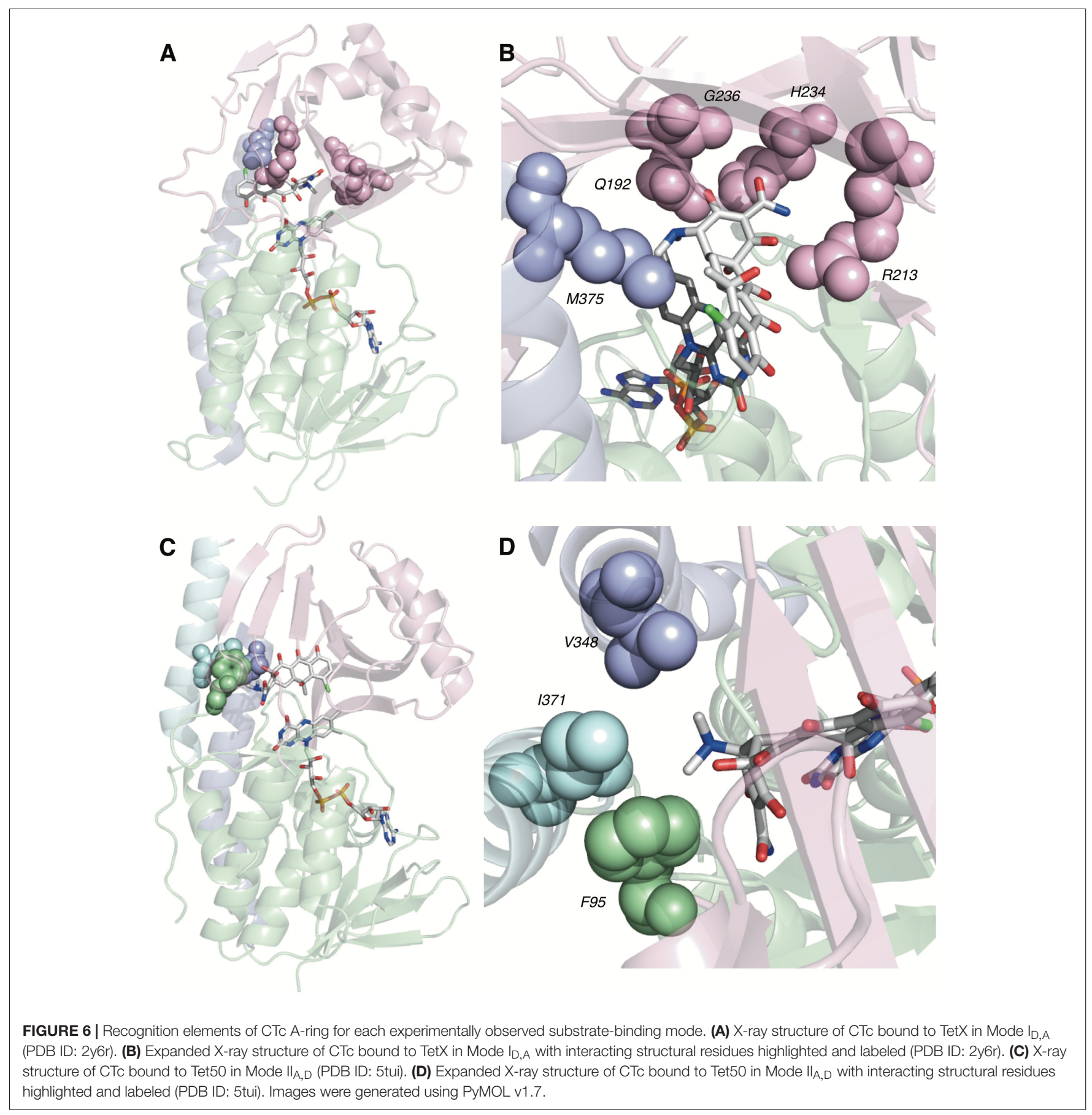

(Figures 6A,B). While a number of hydrophobic residues in the substrate-binding domain also interact with the $\mathrm{C}$ - and D-rings of the enzyme-bound CTc (Volkers et al., 2011), the open cavity near the substrate loading channel of the FAD-IN conformer of TetX allows mostly weak interactions with the D-ring - which can also associate with readily available solvent molecules. In contrast, as shown in Figure 6C, CTc binds to Tet50 in mode $\mathrm{II}_{\mathrm{A}, \mathrm{D}}$ (FAD-IN conformer shown), where several van der Waals interactions between the C-terminal stabilizing alpha-helix, the second C-terminal "gatekeeper" helix, and a residue of the lower FAD-binding domain interact with the $N, N^{\prime}$-(dimethyl)amino substituent of the now "west" tetracycline A-ring. The expanded view of the mode $\mathrm{II}_{\mathrm{A}, \mathrm{D}}$ in Figure $6 \mathrm{D}$ highlights the important contribution of the second C-terminal "gatekeeper" helix which is present in members of the tetracycline destructase family of enzymes but noticeably absent in TetX - to substrate recognition and accommodation. In turn, the substantial differences in experimentally observed substrate-binding modes for CTc-bound TetX and Tet50 account for the variability in 
tetracycline degradation profiles observed for both enzymes, as the proximities of enzyme-reactive functional groups to the C4a-center of the FAD cofactor directly influence the nature of potential degradation cascades (Forsberg et al., 2015; Park et al., 2017).

\section{MECHANISMS OF TETRACYCLINE OXIDATION}

\section{Oxidative "Soft" Spots on the Tetracycline Scaffold}

Due to the unstable nature of tetracyclines to light (Moore et al., 1983; Halling-Sorensen et al., 2002; Fuoco, 2015), heat (Nguyen et al., 2015), and acid or base (Yuen and Sokoloski, 1977), the enzymatic and non-enzymatic degradation profiles of tetracycline antibiotics are complex. The mixtures of products resulting from tetracycline oxidation are likely responsible for the distinct brown colored growth phenotype of E. coli expressing tetracycline destructases (Speer and Salyers, 1989; Yang et al., 2004; Forsberg et al., 2015). However, because the spatial orientation of the substrate in relation to the reactive C4a-peroxyflavin is fundamentally important to the mechanism of oxidation and tetracycline degradation, the experimentally observed binding modes of CTc bound to TetX and Tet50 can be used to identify potential, binding mode-specific, oxidative "soft" spots on the tetracycline scaffold. These oxidative "soft" spots can then be used as starting points to propose potential non-enzymatic degradation cascades to explain experimentally observed degradation profiles (Yang et al., 2004; Forsberg et al., 2015; Park et al., 2017).

For CTc bound in mode $\mathrm{I}_{\mathrm{D}, \mathrm{A}}$, the proposed potential oxidative sites on CTc are the C11a-enol- and C12-carbonyl-carbon centers, at distances of 5.8 and $5.0 \AA$, respectively, from the C4a-position on the FAD cofactor (as determined from the $\mathrm{X}$-ray crystal structure of CTc bound to TetX, Figure 7). This is consistent with the enzymatic hydroxylation of the C11acenter of oxytetracycline by TetX reported by Wright and coworkers in 2004, where acid-stabilizing hemiketal formation of the enzymatic degradation product allowed the authors to isolate and fully characterize the intermediate (see Figure 3, vide supra). For CTc bound in mode $\mathrm{II}_{\mathrm{A}, \mathrm{D}}$, where the A-ring is most accessible to $\mathrm{C} 4 \mathrm{a}$-peroxyflavin oxidation, the proposed potential oxidative sites on CTc are the C1-carbonyl, C2enol, and C3-carbon centers at distances of 7.4, 6.7, and $6.1 \AA$, respectively (as determined from the X-ray crystal structure of CTc bound to Tet50, Figure 7). It appears that the tetracycline substrate is merely a victim of fate and the oxidative "soft spot" that aligns closest to the C4a-peroxyflavin will be oxidized. Properly defining the distance constraints between flavin-C4a and oxidation sites will enable some predictive capacity. A similar oxidative "soft spot" has been reported for the rifamycin monooxygenase (Rox) that hydroxylates the C2 position of the hydroxynaphthol leading to formation of a 1,2-naphthoquinone (Koteva et al., 2018; Liu et al., 2018).

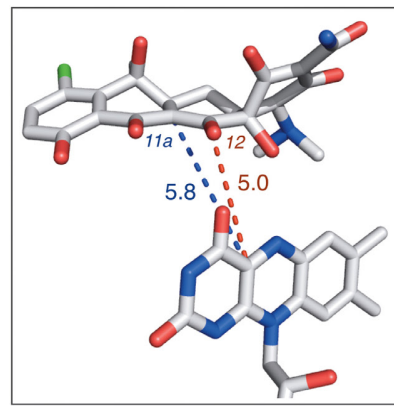

Mode $I_{D, A}$ Substrate Binding TetX $\cdot C T C$
Mode $I_{A, D}$ Substrate Binding Tet50 $\cdot$ CTc

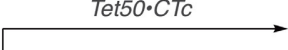

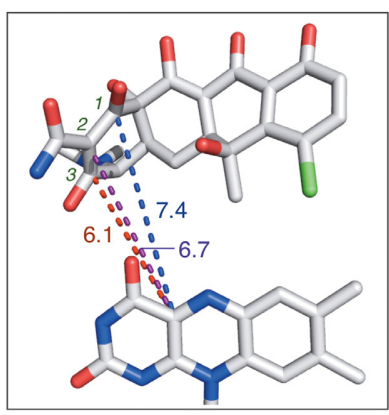
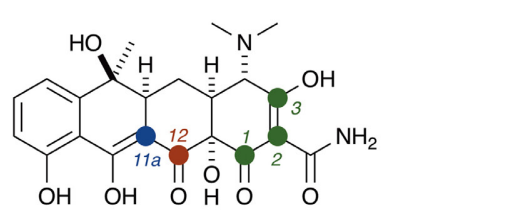

\section{Tet55}
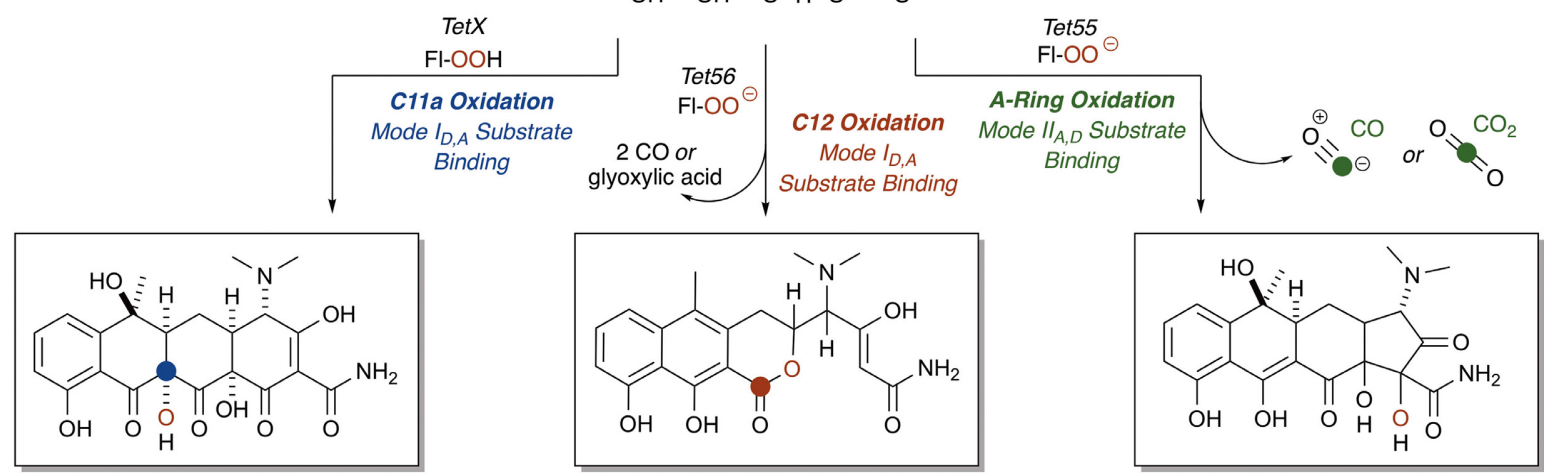

FIGURE 7 | Victim of fate: the site of tetracycline oxidation is determined by binding mode and distance from flavin-C4a. Bond distances to reactive centers on CTC bound to TetX in Mode ID,A (PDB ID: 2y6r) and CTc bound to Tet50 in Mode IIA,D (PDB ID: 5tui) were determined in PyMOL from the corresponding PDB files. Images of FAD were generated using PyMOL v1.7. 
In the rifamycin-Rox structure $\mathrm{C} 2$ is reported to be $4.7 \AA$ from flavin-C4a.

\section{Oxidation Initiates a Cascade of Chemistry}

The highly conjugated nature of the tetracycline antibiotics enables chemical communication across the entire 4-ring structure, which - in turn - can result in a variety of non-enzyme catalyzed rearrangement cascades following the enzymatic oxidation of tetracycline substrates. Indeed, the complex nature of the enzymatic degradation profiles of tetracycline antibiotics and instability of oxidized degradation products implies that non-enzymatic cascade reactions must occur spontaneously in solution to result in a decrease of observed enzymatic degradation product. While the primary enzymatic degradation product of TetX monohydroxylation of oxytetracycline has been observed (Yang et al., 2004), several degradation cascades have been proposed to explain LCMS-observed degradation product formation resulting from the tetracycline destructase-mediated enzymatic oxidation of binding-mode-specific reactive "soft spots" on tetracycline scaffolds (Forsberg et al., 2015; Park et al., 2017).

The nucleophilic or electrophilic nature of the intermediate C4a-peroxyflavin (pKa 7-8; Favaudon, 1977; Kemal et al., 1977) within the enzyme active site can be ambiguous across class A FMO enzymes and is largely dependent upon protonation state (protonated C4a-peroxyflavin = electrophilic species; deprotonated C4a-peroxyflavin = nucleophilic species; Massey, 1994; Montersino and Berkel, 2013; Huijbers et al., 2014). The majority act as electrophiles in the hydroxylation of electron-rich aromatic rings (Wierenga et al., 1979), but BaeyerVilliger chemistry has been observed when the substrate is an electrophilic carbonyl (Ryerson et al., 1982; Schwab et al., 1983; Walsh and Wencewicz, 2013). Thus, for the Tet56-mediated degradation of tetracycline resulting in the formation of major ion $[\mathrm{M}+\mathrm{H}]^{+} 387.1556$, it is proposed that a nucleophilic C4aflavinperoxide can add to the $\mathrm{C} 12$-ketone of tetracycline to form a transient, tetrahedral intermediate (Figure 8; Forsberg et al., 2015). This intermediate can undergo a Baeyer-Villiger-type ring expansion via a 1,2-alkyl-shift to eliminate the C4a-hydroxyflavin and provide an intermediate ester, which upon hemiketal collapse and rearomatization of the former C-ring can provide a naphthyl-substituted cyclohex-4-en-1,2-dione intermediate. Alternatively, the same tetrahedral intermediate can undergo a Grob fragmentation, followed by C-ring aromatization, to arrive at the same naphthyl-substituted cyclohex-4-en-1,2-dione intermediate. This cyclohex-4-en-1,2-dione can then tautomerize and undergo a retro[4+2]-cycloaddition to eliminate either two equivalents of carbon monoxide (CO) or one equivalent of transient oxoketene - that, upon hydrolysis, would provide an equivalent of glyoxylic acid - to afford the proposed degradation product as the naphthylic acid $([\mathrm{M}+\mathrm{H}]+m / z=387.1556)$. Upon Michael addition and enol tautomerization, the naphthylic acid intermediate can provide the corresponding lactone $([\mathrm{M}+\mathrm{H}]+$ $m / z=387.1556)$.

Correspondingly, for the Tet55-mediated degradation of tetracycline resulting in the formation of major ion $[\mathrm{M}+\mathrm{H}]^{+}$ 467.1216 , it is proposed that the nucleophilic addition of the C4a-flavinperoxide to either the $\mathrm{C} 1$ - or $\mathrm{C} 3$-positions of the tetracycline substrate can result in the formation of two, rapidly interconverting epoxide species (Figure 9;
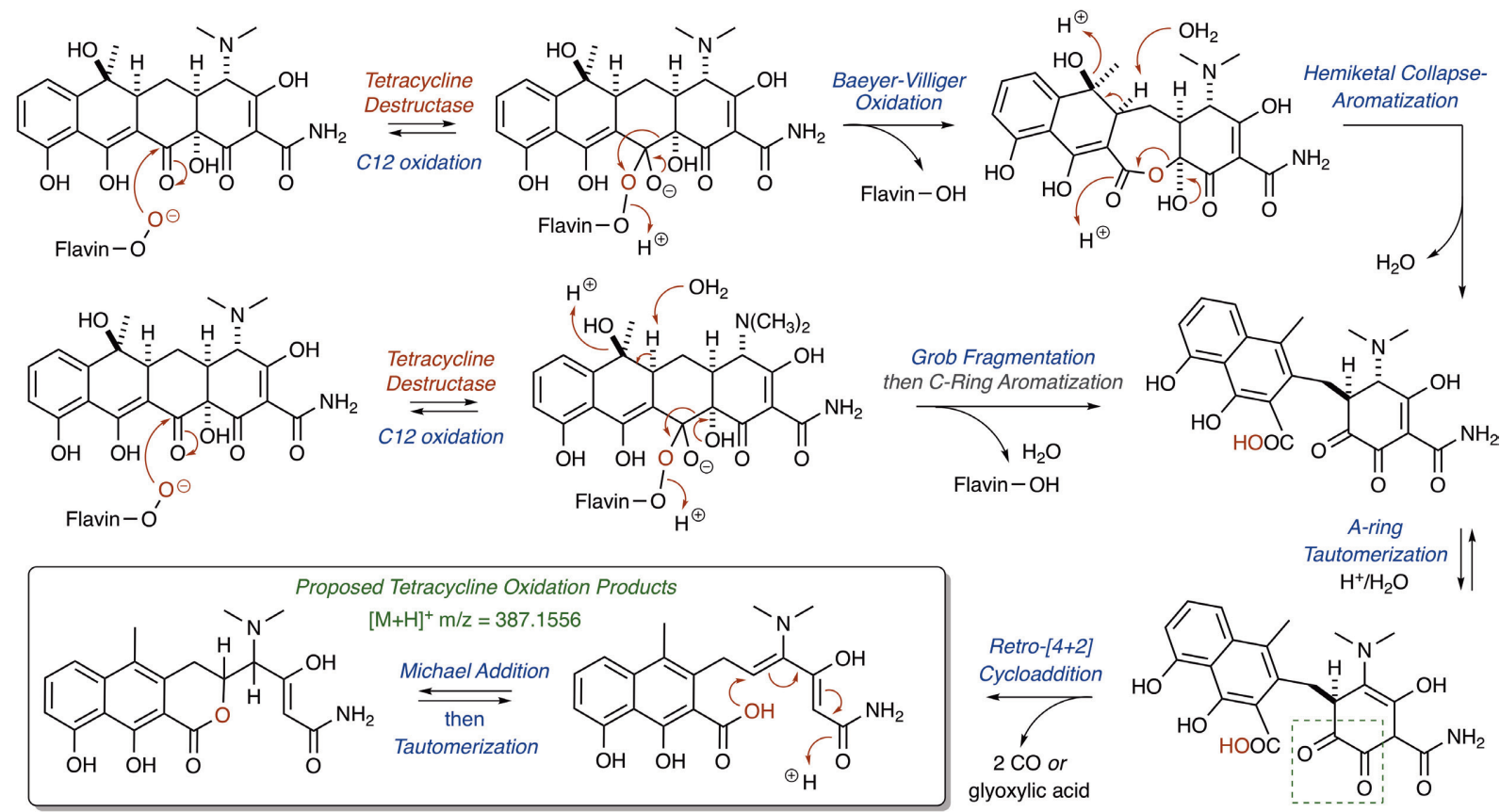

FIGURE 8 | Cascade reactions leading to tetracycline degradation products from enzymatic C12-oxidation of mode $\mathrm{I}_{\mathrm{D}, \mathrm{A}}$-bound tetracycline. 


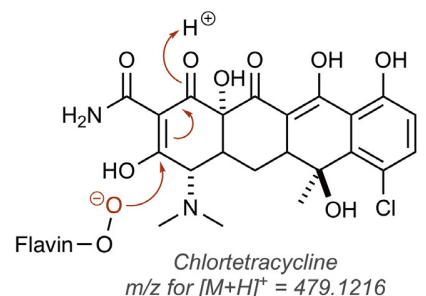

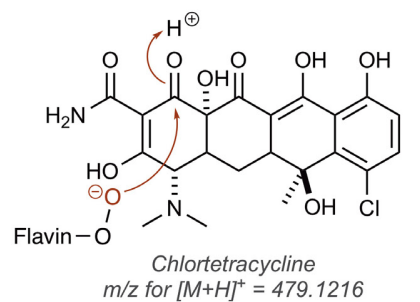
$\mathrm{m} / \mathrm{z}$ for $[M+H]^{+}=479.1216$

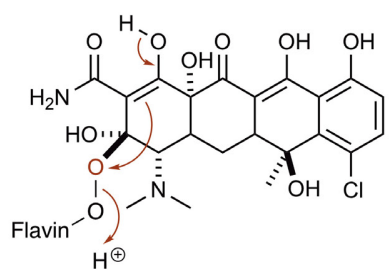

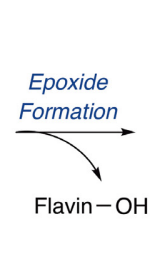

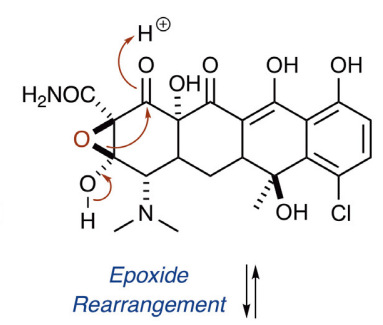

Tetracycline Destructase $\underset{\text { C1 oxidation }}{\rightleftarrows}$

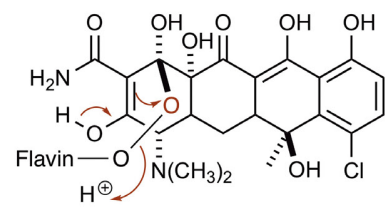
$\stackrel{\substack{\text { Epoxide } \\ \text { Formation }}}{\longrightarrow}$

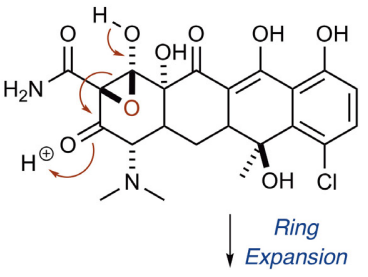<smiles>C[13CH2]CC1(C(N)=O)CC2C(O)=C(O)C3=C(O)c4c(O)ccc(Cl)c4[C@@](C)(O)C3CC2[C@H](N(C)C)C1=O</smiles><smiles>O=CC1CCCCC1</smiles>

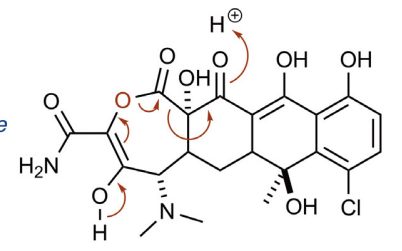<smiles>CN(C)[C@H]1C(=O)C(O)(C(N)=O)[C@@]2(O)C(=O)C3=C(O)c4c(O)ccc(Cl)c4[C@@](C)(O)C3CC12</smiles>

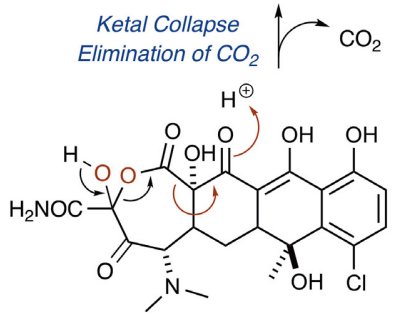

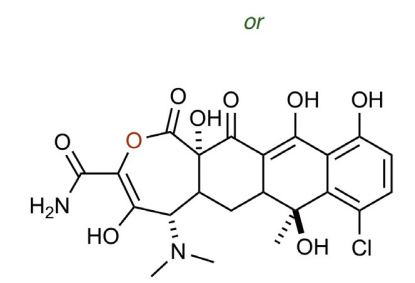

FIGURE 9 | Cascade reactions leading to tetracycline degradation products from enzymatic C1- or C3-oxidation of mode $\|_{A, D}$-bound tetracycline.

Park et al., 2017). These species can undergo a ring-expansion via collapse of the hemiketal-epoxide to provide an intermediate lactone, which upon elimination of one equivalent of $\mathrm{CO}$ and subsequent intramolecular 5-(enol-exo)-exo-trig cyclization of the resultant enol-containing alpha-ketoamide, could be converted to the proposed degradation product $\left([\mathrm{M}+\mathrm{H}]^{+}\right.$ 467.1216). Alternatively, the intermediate lactone could undergo a second enol oxidation, followed by ketal collapse and extrusion of carbon dioxide $\left(\mathrm{CO}_{2}\right)$, to provide the same enol-containing alpha-ketoamide, which after intramolecular 5-(enol-exo)-exotrig cyclization provides the corresponding degradation product.

Alternatively, hydroxylation of C2 with an electrophilic C4aperoxyflavin would initiate a cascade resulting in the same degradation product (Figure 10; $[\mathrm{M}+\mathrm{H}]^{+}$467.1216). A similar hydroxylation of $\mathrm{C} 2$ in mithramycin biosynthesis initiates a ring opening cascade to provide the bioactive form of the DNA minor groove-binding molecule (Gibson et al., 2005). While the precise degradation products remain unknown for both the enzymatic oxidation and the following non-enzymatic degradation cascade, these mechanistic proposals may serve as useful models as more information becomes available en route to the elucidation of the enzymatic degradation of tetracycline antibiotics (Yang et al., 2004; Forsberg et al., 2015; Ghosh et al., 2015; Park et al.,
2017). It is noteworthy that a similar cascade event takes place for the Rox-mediated inactivation of rifamycin where oxidation of the C2 position of the hydroxynaphthalene leads to ring opening of the macrolactam and subsequent linearization of rifamycin (Koteva et al., 2018; Liu et al., 2018). A detailed understanding of enzymatic and non-enzymatic degradation cascades for tetracycline and other antibiotics is critical for designing future generations of molecules that overcome these resistance mechanisms and diagnostic tools to detect active antibiotic-inactivating enzymes in clinical samples. In fact, the degradation mechanisms of beta-lactam antibiotics by betalactamase enzymes were fundamentally important to the design of fluorogenic and chromogenic probes used in clinical diagnostic applications (O'Callaghan et al., 1972; Yu et al., 2012; Ghavami et al., 2015).

\section{TETRACYCLINE DESTRUCTASE INHIBITORS, AN ADJUVANT APPROACH}

\section{Antibiotic Destructase Inhibitors}

There are two clinically proven approaches to overcoming resistance by antibiotic destructases: (1) modification of the 


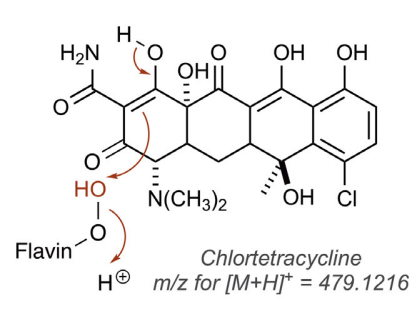

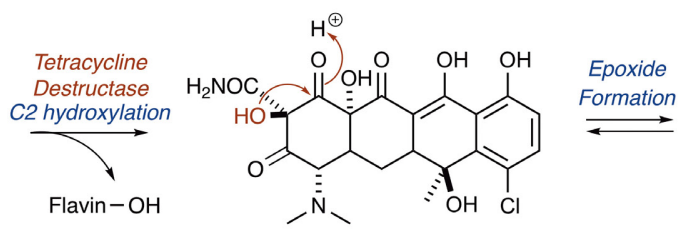

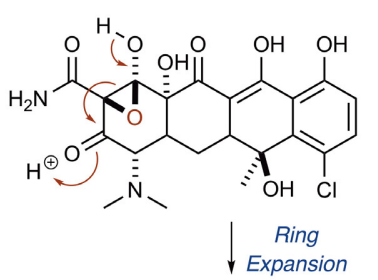

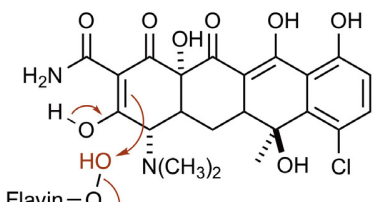
Flavin-O

$\mathrm{H}^{\oplus} \quad$ Chlortetracycline $\mathrm{m} / \mathrm{z}$ for $[\mathrm{M}+\mathrm{H}]^{+}=479.1216$<smiles>CN(C)[C@H]1C(O)=C(C(N)=O)OC(=O)[C@]2(C)C(=O)C3=C(O)c4c(O)ccc(Cl)c4[C@@](C)(O)C3C[C@H]12</smiles>

FIGURE 10 | Alternative mechanistic pathway leading to formation of the ring contracted degradation product $\left([\mathrm{M}+\mathrm{H}]^{+} 467.1216\right)$ initiated by hydroxylation of $\mathrm{C} 2$.
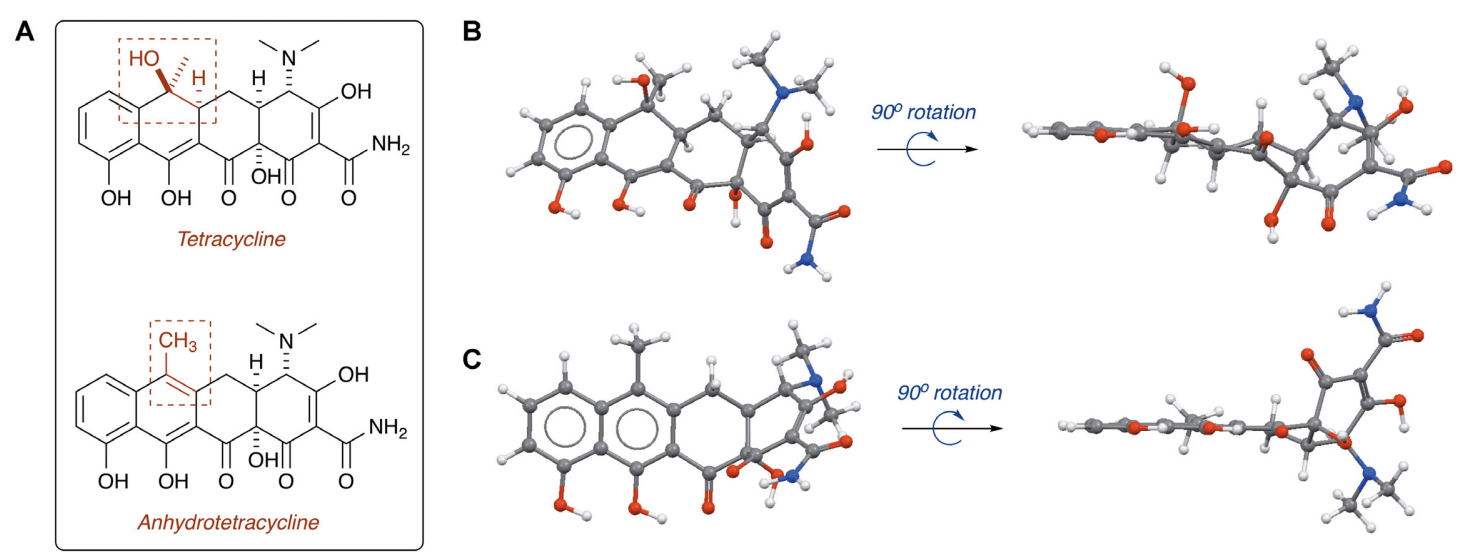

FIGURE 11 | (A) Structures of tetracycline (top) and anhydrotetracycline (bottom). Conformation of tetracycline (B) and anhydrotetracycline (C) as viewed from face and edge of the tetracyclic core. 3D structures of tetracycline and anhydrotetracycline in panels (B) and (C) were energy minimized using Spartan and images were generated using Mercury software v3.10.

antibiotic structure in a manner that prevents covalent modification (i.e., successive generations of beta-lactams) (Fisher et al., 2005); (2) co-administration of an adjuvant that inhibits production and/or catalytic activity of antibiotic destructases (i.e., beta-lactam/beta-lactamase inhibitor combinations) (Bush, 2018). Modern beta-lactam antibiotics are now fifth generation scaffold iterations, and it is rare to push new beta-lactams into the clinic without co-administration of a beta-lactamase inhibitor. The first beta-lactamase inhibitors, such as clavulanic acid isolated from Streptomyces clavuligerus, were found to be beta-lactams like the parent antibiotic (Reading and Cole, 1977). Nature seems to have invented this adjuvant approach long before medicinal chemists ever proposed the idea. In addition to clavulanic acid, $S$. clavuligerus also produces the cephalosporin antibiotic cephamycin. The biosynthetic genes for both clavulanic acid and cephamycin are colocalized in a "super cluster" operon, resulting in simultaneous production of the antibiotic and adjuvant to ensure efficacy against betalactamase-producing competitors (Ward and Hodgson, 1993). It is conceivable that tetracycline producers can also biosynthesize tetracycline destructase inhibitors to protect the tetracycline antibiotic, though evidence of which has yet to be discovered.

\section{Anhydrotetracycline - The First Tetracycline Destructase Inhibitor}

Tetracycline producers readily excrete analogs and shunt products during tetracycline biosynthesis (Pickens and Tang, 2010; Wang et al., 2013). One intermediate and shunt product in tetracycline biosynthesis, anhydrotetracycline, was found to be a poor substrate for the tetracycline destructases (Forsberg et al., 2015; Park et al., 2017). Only TetX was able to oxidize anhydrotetracycline, albeit very slowly, suggesting that tetracycline destructases can still bind anhydrotetracycline in the substrate-binding domain despite the subtle structural differences compared to the parent tetracycline (Figure 11). Dehydration of the tetracycline scaffold at C6 provides the more hydrophobic anhydrotetracycline with a flattened naphthalene C,D-ring system and some conformational changes 
in the A,B-rings. Despite the subtle structural differences, tetracycline and anhydrotetracycline show remarkably different biological activity. Tetracyclines are potent ribosome inhibitors and have an overall bacteriostatic effect on cells (Wilson, 2009). Anhydrotetracyclines are weak ribosome inhibitors and have a bactericidal effect on cells, presumably through membrane depolarization (Rasmussen et al., 1991; Oliva et al., 1992). Anhydrotetracycline was able to rescue the activity of tetracyclines when co-administered in checkerboard antibacterial assays against $E$. coli expressing tetracycline destructases (Park et al., 2017). Furthermore, anhydrotetracycline was shown to be a potent inhibitor of tetracycline destructases in vitro at low micromolar levels. It remains unclear if anhydrotetracycline is acting as a true competitive inhibitor or a competitive (slow) substrate. These initial studies suggest that anhydrotetracycline is a viable tetracycline destructase lead inhibitor and sets the stage for developing tetracycline destructase inhibitors as adjuvants for use in combination therapy with tetracycline antibiotics. This also raises the question as to whether tetracyclineproducing microbes excrete anhydrotetracycline with the tetracycline antibiotic to act synergistically as tetracycline destructase inhibitors and secondary antibiotics with an alternate mode of action (membrane depolarization). Mixtures of tetracycline and tetracycline degradation products, including anhydrotetracycline, have been shown to invert resistance selection and select against tetracycline efflux pumps (Palmer et al., 2010; Chait et al., 2011; Stone et al., 2016). Tetracycline destructases and associated degradation products might play a variety of roles beyond resistance in natural environments, including signaling and control of microbial populations (Yim et al., 2007).

\section{Structural and Mechanistic Basis for Inhibition}

X-ray crystal structures of anhydrotetracycline bound to Tet50 revealed several important distinctions in the binding mode compared to the previously discussed structures of CTc bound to Tet50 and TetX (Figure 12; Park et al., 2017). First, a new binding orientation of the tetracyclic scaffold, Mode $\mathrm{I}_{\mathrm{A}, \mathrm{D}}$, was observed (see Figure 5 for reference). The flattened C,Dring system enables anhydrotetracycline to bind deeper in the substrate-binding domain with the C6-methyl group filling a hydrophobic pocket lined by L198, T207, L205, M222, V181, P296, and Q44. This orientation pushes the flavin "out" and orients the gatekeeper helix so the active site is open to solvent. This binding mode is inaccessible to canonical tetracyclines with methylation and hydroxylation at C6 due to steric clashing. The anhydrotetracycline-stabilized Tet50 conformation is predicted to be catalytically incompetent; however, other binding modes with anhydrotetracycline might be possible based on the observed plasticity of the tetracycline destructases for CTc. Since TetX can slowly oxidize anhydrotetracycline, it seems possible that anhydrotetracyclines can bind in alternate modes similar to CTc that might enable the flavin cofactor to reach the catalytically competent "in" conformation. The gatekeeper helix might be the distinguishing structural feature between TetX and other tetracycline destructases that determines conformational dynamics, substrate plasticity, catalytic efficiency, and susceptibility to inhibition. The structure of anhydrotetracycline bound to Tet50 should serve as a guide for structure-based drug design of improved tetracycline destructase inhibitors.

\section{Mechanistic Model for Catalysis and Inhibition}

Based on the structural (the section "Structural Basis for Tetracycline Inactivation"), mechanistic (the section "Mechanisms of Tetracycline Oxidation"), and inhibition (the section "Tetracycline Destructase Inhibitors, an Adjuvant Approach") studies of the tetracycline destructases, a generic model for the catalytic cycle of tetracycline inactivation and inhibition is proposed (Figure 13). The tetracycline destructase can exist in a resting state with the flavin in the oxidized form (I). As shown for other class A FMOs (Abdelwahab et al., 2016), substrate binding (II) can accelerate flavin reduction by NADPH (III) with the flavin dynamically moving between the FAD-IN and FAD-OUT states. The timing and location of C4a-peroxyflavin formation is unclear, but presumably, to oxidize the substrate, the C4a-peroxyflavin must move to the "in" conformation (IV). If the tetracycline destructase has a gatekeeper helix, then the enzyme active site will be closed when the flavin transitions from "out" to "in" and ultimately is positioned to oxidize the tetracycline substrate (V). Movement of the flavin to the "out" conformation will result in movement of the gatekeeper helix to open the active site and release the tetracycline product to complete the catalytic cycle (VI). The oxidized tetracycline products might be subject to further enzymatic oxidation or non-enzymatic cascade reactions leading to non-antibacterial tetracycline degradation products. Anhydrotetracycline is predicted to competitively bind in the substrate-binding domain, which can lead to formation of a stabilized tetracycline destructase inhibition complex with the flavin cofactor essentially "locked" in the unproductive "out" conformation (VII). Anhydrotetracycline is slowly oxidized by TetX; thus, it is conceivable that net destructase inhibition is achieved by anhydrotetracycline acting as a competitive (slow) substrate (VIII). FMOs are a diverse family of oxidoreductases that perform a staggering array of transformations (Walsh and Wencewicz, 2013). There are still many unanswered questions regarding the timing and mechanism of tetracycline inactivation and tetracycline destructase inhibition that will require further structural, mechanistic, and kinetic studies (Eswaramoorthy et al., 2006; van Berkel et al., 2006; Romero et al., 2018).

\section{FINAL THOUGHTS}

\section{Tetracycline Destructases Represent an Emerging Threat to Next-Generation Tetracyclines}

Antibiotic resistance is a moving target (Wright, 2007). Tetracyclines have kept pace through advancements in scaffold 

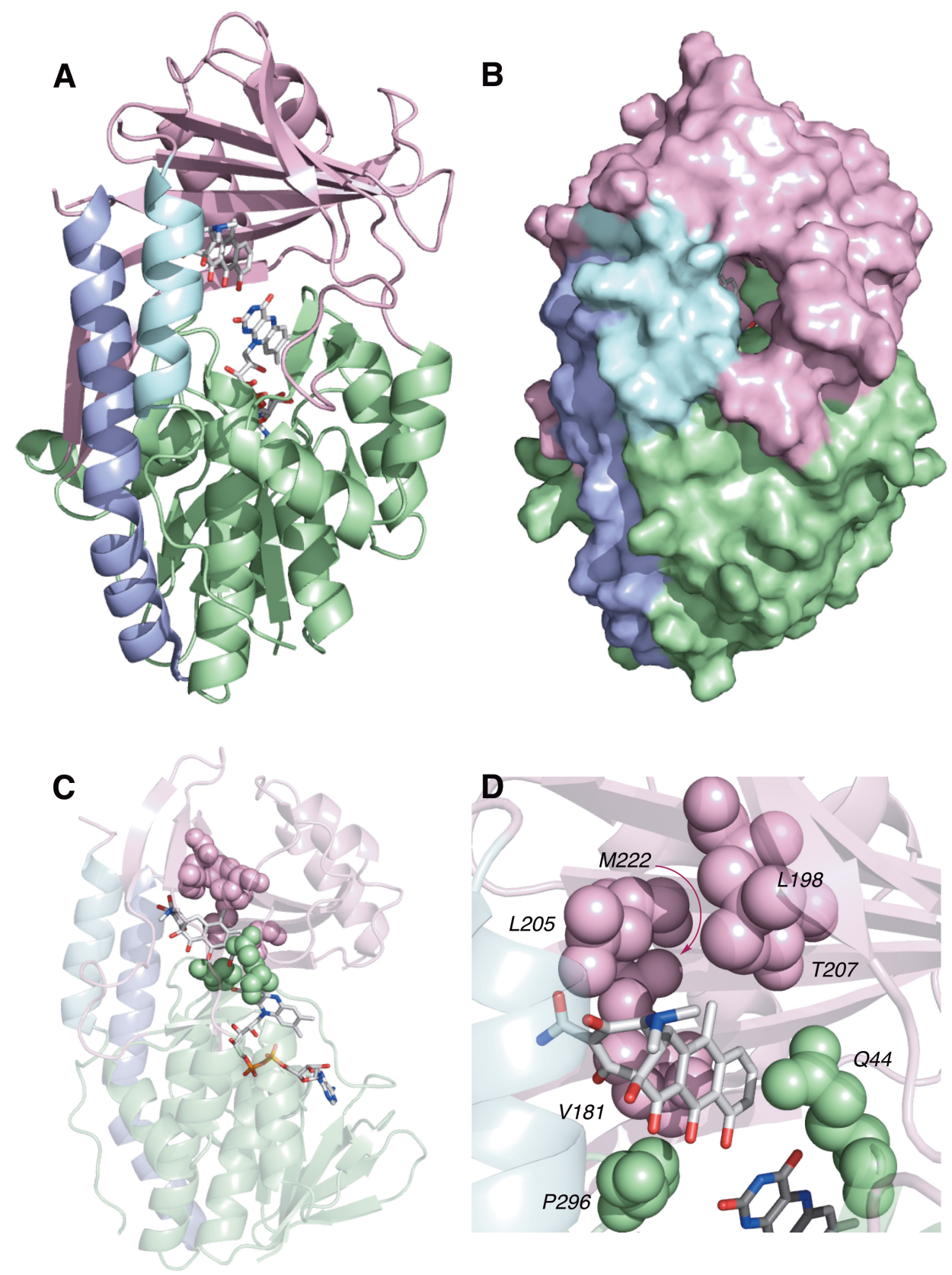

FIGURE 12 | (A) X-ray crystal structure of anhydrotetracycline bound to Tet50 in Mode $\mathrm{I}_{\mathrm{A}, \mathrm{D}}$ (PDB accession number 5 tuf). (B) Surface view of X-ray crystal structure of aTC bound to Tet50. (C) X-ray crystal structure of anhydrotetracycline bound to Tet50 in Mode $I_{A, D}$ with recognition residues highlighted. (D) Expanded X-ray crystal structure of anhydrotetracycline bound to Tet50 in Mode $\mathrm{I}_{\mathrm{A}, \mathrm{D}}$ with recognition residues highlighted and labeled. Images were generated using PyMOL v1.7.

derivatization, semi-synthesis, biosynthesis, and total chemical synthesis (Chopra and Roberts, 2001; Liu and Myers, 2016; Sun and Xiao, 2017). Increased use of third (tigecycline) and fourth generation (eravacycline, omadacycline) tetracyclines that overcome resistance by efflux and ribosome protection threaten to select for new resistance mechanisms. The tetracycline destructases are FMOs that confer resistance to these nextgeneration tetracyclines via covalent inactivation (Moore et al., 2005; Grossman et al., 2012; Sutcliffe et al., 2013;
Volkers et al., 2013). Antibiotic oxidation is an emerging inactivation resistance strategy that has only been observed for one other antibiotic class, the rifamycins (Abdelwahab et al., 2016; Liu et al., 2016, 2018; Koteva et al., 2018). Resistance to rifamycin via enzymatic inactivation is not limited to FMOs; in fact, known rifamycin destructases include FMOs (Abdelwahab et al., 2016; Liu et al., 2016, 2018; Koteva et al., 2018), glycosyltransferases (Spanogiannopoulos et al., 2012), ADP-ribosyltransferases (Baysarowich et al., 


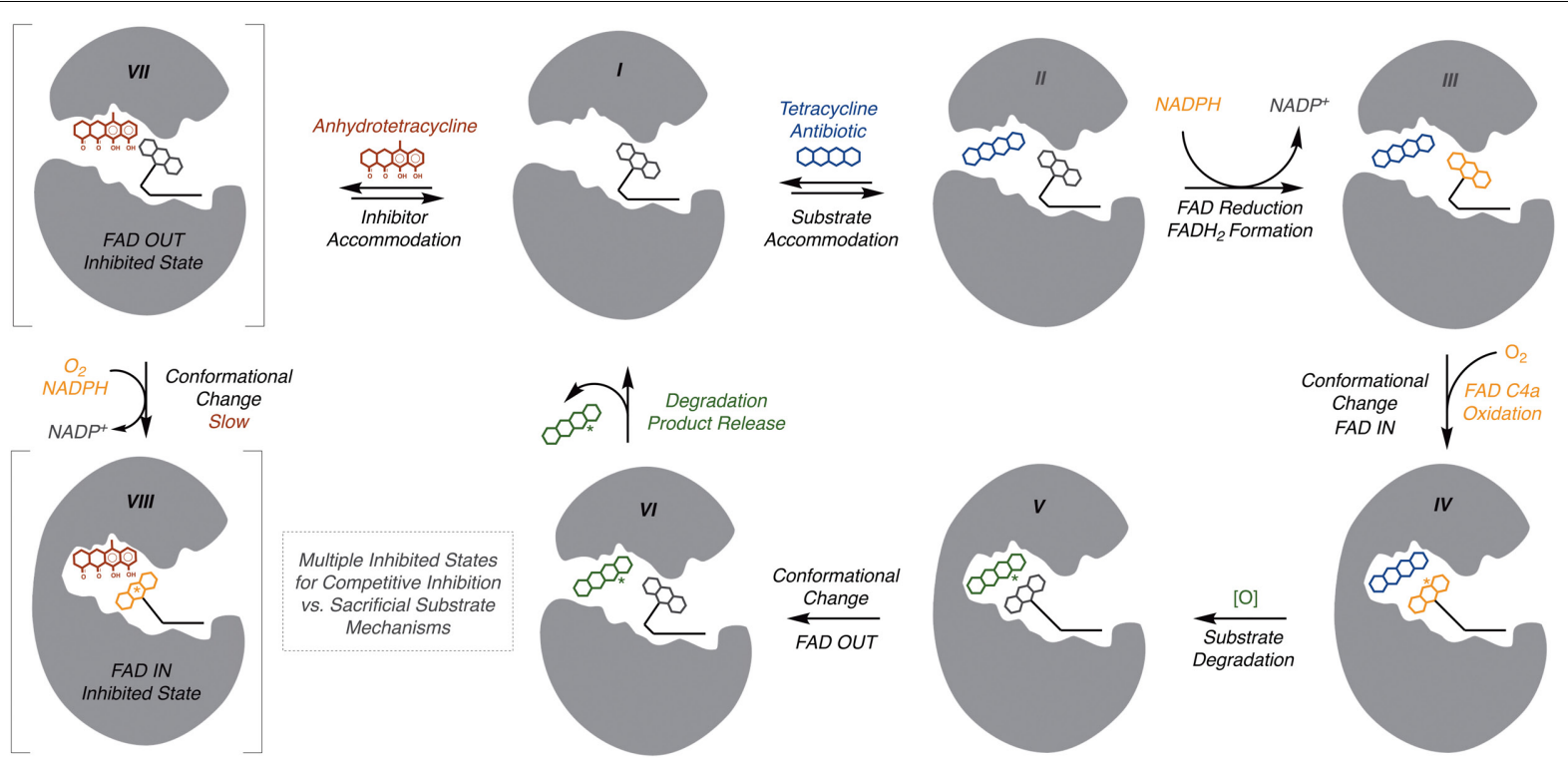

FIGURE 13 | A mechanistic model for the tetracycline destructase catalytic cycle and inhibition by anhydrotetracycline is proposed. (I) Flavin oxidized, open active site; (II) substrate binding, flavin oxidized, open active site; (III) substrate bound, flavin reduced, open active site; (IV) substrate bound with C4a-peroxyflavin in "in" conformation, closed active site; ( $)$ oxidized product bound with C4a-hydroxyflavin in "in" conformation, closed active site; (VI) substrate bound with C4a-hydroxyflavin in "out" conformation, open active site; (VII) inhibitor bound with flavin in "out" conformation, open active site; (VIII) inhibitor bound with C4a-peroxyflavin in "in" conformation, closed active site.

2008), and phosphotransferases (Stogios et al., 2016). Future prospecting for tetracycline ARGs will likely result in the discovery of non-FMO tetracycline destructases. Tetracyclines, and rifamycins, are sensitive to chemical photooxidation; so, it seems appropriate that the first tetracycline destructases, FMOs, exploit this reactivity (Moore et al., 1983). The relevance of FMO tetracycline destructases is presumably limited to aerobic infections due to the strict requirement of molecular oxygen for tetracycline inactivation (Guiney et al., 1984). Historically, tetracyclines have been found to be more effective against aerobic bacteria than anaerobic bacteria (Chow et al., 1975). Thus, acquisition and expression of tetracycline destructase FMO genes will be beneficial for aerobic and facultatively anaerobic pathogens that cause a variety of aerobic infections, including pulmonary, periodontal, skin, and post-surgical wound infections (Chopra and Roberts, 2001).

\section{Functional Prospecting Is Needed to Monitor the Dissemination of Tetracycline Destructase Genes in Human Pathogens}

Tetracycline destructases have emerged on mobile genetic elements in human bacterial pathogens (Leski et al., 2013). It appears urgent to have a management plan for tetracycline destructases in place before a clinical crisis emerges. Functional metagenomics is an effective strategy to monitor the dissemination of tetracycline destructases in hospitals and should be continuously applied to patient samples and clinical isolates (Crofts et al., 2017). Tetracycline destructases, including
TetX, evolved in the presence of countless tetracycline variants in diverse environments and thus gained great substrate plasticity (Forsberg et al., 2015; Park et al., 2017). This explains the ability of TetX to oxidize never-before-seen synthetic tetracyclines, including the clinical antibiotics tigecycline and eravacycline, through flexibility in substrate-binding mode that allows for accommodation of tetracyclines with bulky D-ring substituents (Figure 14; Grossman et al., 2012; Sutcliffe et al., 2013). This same type of substrate plasticity has been well documented for the beta-lactamases and carries the risk of causing pan-resistance to an entire drug class (Bush and Jacoby, 2010). A recent study showed that random mutagenesis of the tet $X$ gene readily provided TetX variants with significantly improved activity toward tigecycline inactivation (Linkevicius et al., 2016). This suggests that tet $X$ is poised to emerge as a primary resistance mechanism under tigecycline selective pressure. Similar to tigecycline, fourth generation molecules like eravacycline and omadacycline possess bulky D-ring substituents that are accommodated and solvent exposed by the constitutively open TetX active site (Figure 14). Comprehensive study of the functional evolution and evolvability of the tetracycline destructases is merited to anticipate future enzyme variants that might emerge in human pathogens (Brandt et al., 2017; Crofts et al., 2017; Pawlowski et al., 2018). It will be interesting to look to tetracycline biosynthetic pathways for FMOs that introduce scaffold oxidations to see if these can undergo gain-of-function as destructases, which might point toward a pathway of evolution for the tetracycline destructases (Gibson et al., 2005; Pickens and Tang, 2010; Walsh and Wencewicz, 2013; Wang et al., 2013). 

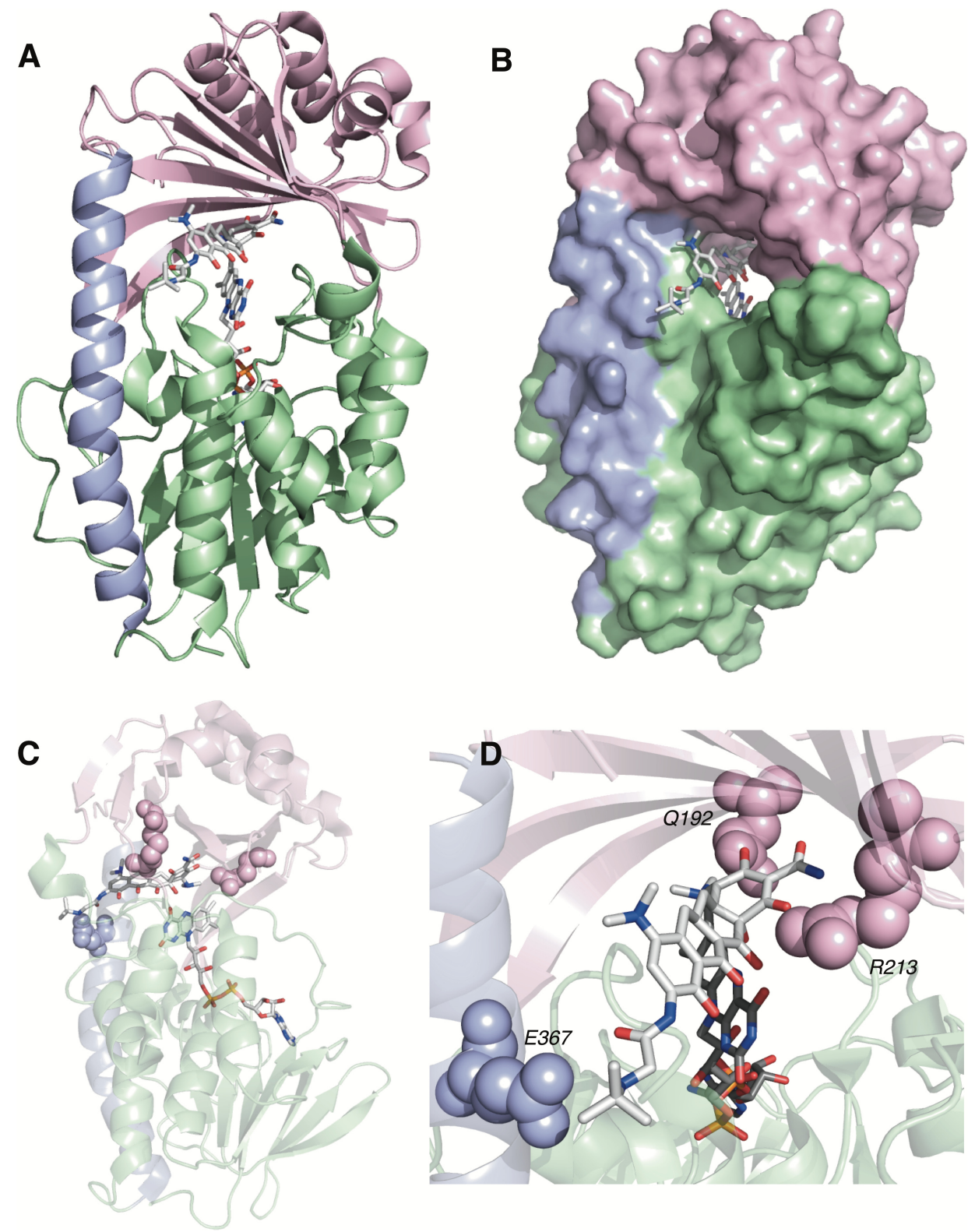

FIGURE 14 | (A) X-ray structure of tigecycline bound to TetX in Mode $\mathrm{I}_{D, A}$ (PDB accession number 4a6n). (B) Surface view of X-ray structure of tigecycline bound to TetX in Mode $I_{D, A}$. (C) X-ray structure of tigecycline bound to TetX in Mode $I_{D, A}$ with relevant substrate recognition interactions highlighted. (D) Expanded X-ray structure of tigecycline bound to TetX in Mode $\mathrm{I}_{\mathrm{D}, \mathrm{A}}$ with relevant substrate recognition interactions highlighted for the A-ring (Q192, R213) and the D-ring $\mathrm{N}$-t-butyl-glycylamide substituent (E367). Electron density for the C2-carboxamide bond was missing in the PDB file 4a6n. The C2-carboxamide bond was added using the create bond function in PyMOL. Images were generated using PyMOL v1.7.

\section{Inhibitors Are Needed as Tetracycline Adjuvants for Future Combination Therapy}

Mechanistic and kinetic evaluation of tetracycline destructases have revealed an impressive capacity for substrate oxidation at diverse scaffold positions (Figure 7). Further studies will be needed to map oxidative soft spots to guide the synthesis of next-generation tetracyclines that block oxidation by tetracycline destructases and maintain high affinity for the bacterial ribosome.
The high degree of substrate plasticity of the tetracycline destructases suggests that inhibitors will likely be needed as adjuvants for combination therapies with tetracycline antibiotics. The history of beta-lactamases tells us that scaffold iteration is not enough, and it would be prudent to have inhibitors in hand before tetracycline destructases become a widespread clinical resistance mechanism. Anhydrotetracycline has emerged as the first tetracycline destructase inhibitor and shows potential to be a pan-destructase inhibitor. TetX can slowly oxidize 
anhydrotetracycline; thus, models as both a competitive inhibitor and a competitive sacrificial substrate should be applied. Most beta-lactamase inhibitors are mechanism-based and act as both sacrificial substrates and covalent inhibitors, providing clinical evidence that this model of destructase inhibition is viable. The crystal structure of anhydrotetracycline bound to Tet50 in a novel binding mode that presumably locks the flavin cofactor in the "out" conformation is a good starting point for structure-based drug design (Figure 12; Park et al., 2017). Anhydrotetracyclines do have antibacterial activity as membrane disruptors and are capable of cell permeation even at subMIC levels relevant for tetracycline destructase inhibition when used in combination with tetracycline antibiotics (Rasmussen et al., 1991). In addition to anhydrotetracycline, a variety of inhibitor scaffolds would be beneficial, as history from betalactamases tells us that multiple inhibitor types will be needed to keep pace with the constantly evolving destructases (Drawz and Bonomo, 2010; Bush, 2018). For antibiotic resistance it is not a question of if, but when it will become a clinical problem, which begs the question: When will we take notice? Given the historical precedence for enzymatic antibiotic inactivation mechanisms to dominate resistance landscapes, it is conceivable that all next-generation tetracyclines will need to be co-administered with a tetracycline destructase inhibitor potentially in our lifetime. Therefore, a proactive approach to developing next-generation tetracyclines and tetracycline destructase inhibitors is the prudent solution to avoiding a clinical crisis ... for now.

\section{REFERENCES}

Abdelwahab, H., Martin Del Campo, J. S., Dai, Y., Adly, C., El-Sohaimy, S., and Sobrado, P. (2016). Mechanism of rifampicin inactivation in Nocardia farcinica. PLoS One 11:e0162578. doi: 10.1371/journal.pone.0162578

Aminov, R. I. (2009). The role of antibiotics and antibiotic resistance in nature. Environ. Microbiol. 11, 2970-2988. doi: 10.1111/j.1462-2920.2009. 01972.x

Aminov, R. I. (2013). Evolution in action: dissemination of tet(X) into pathogenic microbiota. Front. Microbiol. 4:192. doi: 10.3389/fmicb.2013. 00192

Baysarowich, J., Koteva, K., Hughes, D. W., Ejim, L., Griffiths, E., Zhang, K., et al. (2008). Rifamycin antibiotic resistance by ADP-ribosylation: structure and diversity of Arr. Proc. Natl. Acad. Sci. U.S.A. 105, 4886-4891. doi: 10.1073/pnas. 0711939105

Bolam, D. N., Roberts, S., Proctor, M. R., Turkenburg, J. P., Dodson, E. J., Martinez-Fleites, C., et al. (2007). The crystal structure of two macrolide glycosyltransferases provides a blueprint for host cell antibiotic immunity. Proc. Natl. Acad. Sci. U.S.A. 104, 5336-5341. doi: 10.1073/pnas.060789 7104

Botterman, J., Gosselé, V., Thoen, C., and Lauwereys, M. (1991). Characterization of phosphinothricin acetyltransferase and C-terminal enzymatically active fusion proteins. Gene 102, 33-37. doi: 10.1016/0378-1119(91)90534-1

Brandt, C., Braun, S. D., Stein, C., Slickers, P., Ehricht, R., Pletz, M. W., et al. (2017). In silico serine $\beta$-lactamases analysis reveals a huge potential resistome in environmental and pathogenic species. Sci. Rep. 7:43232. doi: 10.1038/ srep43232

Brodersen, D. E., Clemons, W. M. Jr., Carter, A. P., Morgan-Warren, R. J., Wimberly, B. T., and Ramakrishnan, V. (2000). The structural basis for the action of the antibiotics tetracycline, pactamycin, and hygromycin $\mathrm{B}$ on the $30 \mathrm{~S}$ ribosomal subunit. Cell 103, 1143-1154. doi: 10.1016/S0092-8674(00)00216-6

\section{AUTHOR CONTRIBUTIONS}

JM and TW wrote the manuscript and prepared the figures.

\section{FUNDING}

TW and JM acknowledge the National Institute of Allergy and Infectious Diseases, National Institutes of Health, grant R01 123394 for supporting our work on tetracycline-inactivating enzymes.

\section{ACKNOWLEDGMENTS}

A special acknowledgment is given to the tetracycline destructase research team at Washington University in St. Louis including Prof. Gautam Dantas (School of Medicine), Prof. Niraj Tolia (School of Medicine), Prof. Timothy Wencewicz (Department of Chemistry), Dr. Kevin Forsberg (Dantas Lab; currently at Fred Hutch Cancer Research Center), Dr. Jooyoung Park (Tolia Lab; currently at University of Washington), Dr. Jana Markley (Wencewicz Lab), Dr. Hirdesh Kumar (Tolia Lab), Dr. Jie Sun (Dantas Lab; currently at North Carolina State University), Andrew Gasparrini (Dantas Lab), Margaret Reck (Wencewicz Lab; currently at Carboline, St. Louis, MO, United States), Chanez Symister (Wencewicz Lab), Luting Fang (Wencewicz Lab), and Tayte Campbell (Dantas Lab).

Brown, E. D., and Wright, G. D. (2016). Antibacterial drug discovery in the resistance era. Nature 529, 336-343. doi: 10.1038/nature17042

Burdett, V. (1986). Streptococcal tetracycline resistance mediated at the level of protein synthesis. J. Bacteriol. 165, 564-569. doi: 10.1128/jb.165.2.564-569.1986

Burdett, V. (1991). Purification and characterization of Tet(M), a protein that renders ribosomes resistant to tetracycline. J. Biol. Chem. 266, 2872-2877.

Burdett, V. (1996). Tet(M)-promoted release of tetracycline from ribosomes is GTP dependent. J. Bacteriol. 178, 3246-3251. doi: 10.1128/jb.178.11.3246-3251.1996

Burdett, V., Inamine, J., and Rajagopalan, S. (1982). Heterogeneity of tetracycline resistance determinants in Streptococcus. J. Bacteriol. 149, 995-1004.

Bush, K. (2018). Game Changers: new $\beta$-lactamase inhibitor combinations targeting antibiotic resistance in gram-negative bacteria. ACS Infect. Dis. 4, 84-87. doi: 10.1021/acsinfecdis.7b00243

Bush, K., and Jacoby, G. A. (2010). Updated functional classification of $\beta$-lactamases. Antimicrob. Agents Chemother. 54, 969-976. doi: 10.1128/AAC. 01009-09

Chait, R., Vetsigian, K., and Kishony, R. (2011). What counters antibiotic resistance in nature? Nat. Chem. Biol. 8, 2-5. doi: 10.1038/nchembio.745

Charest, M. G., Lerner, C. D., Brubaker, J. D., Siegel, D. R., and Myers, A. G. (2005). A convergent enantioselective route to structurally diverse 6-deoxytetracycline antibiotics. Science 308, 395-398. doi: 10.1126/science.1109755

Chopra, I., and Roberts, M. (2001). Tetracycline antibiotics: mode of action, applications, molecular biology, and epidemiology of bacterial resistance. Microbiol. Mol. Biol. Rev. 65, 232-260. doi: 10.1128/mmbr.65.2.232-260.2001

Chow, A. W., Patten, V., and Guze, L. B. (1975). Comparative susceptibility of anaerobic bacteria to minocycline, doxycycline, and tetracycline. Antimicrob. Agents Chemother. 7, 46-49. doi: 10.1128/AAC.7.1.46

Cocozaki, A. I., Altman, R. B., Huang, J., Buurman, E. T., Kazmirski, S. L., Doig, P., et al. (2016). Resistance mutations generate divergent antibiotic susceptibility profiles against translation inhibitors. Proc. Natl. Acad. Sci. U.S.A. 113, 8188-8193. doi: 10.1073/pnas.1605127113 
Cohen, S. P., McMurry, L. M., and Levy, S. B. (1988). marA locus causes decreased expression of $\mathrm{OmpF}$ porin in multiple-antibiotic-resistant (Mar) mutants of Escherichia coli. J. Bacteriol. 170, 5416-5422. doi: 10.1128/jb.170.12.5416-5422

Connell, S. R., Tracz, D. M., Nierhaus, K. H., and Taylor, D. E. (2003). Ribosomal protection proteins and their mechanism of tetracycline resistance. Antimicrob. Agents Chemother. 47, 3675-3681. doi: 10.1128/aac.47.12.3675-3681.2003

Crofts, T. S., Gasparrini, A. J., and Dantas, G. (2017). Next-generation approaches to understand and combat the antibiotic resistome. Nat. Rev. Microbiol. 15, 422-434. doi: 10.1038/nrmicro.2017.28

Davies, J. (1994). Inactivation of antibiotics and the dissemination of resistance genes. Science 264, 375-382. doi: 10.1126/science.8153624

Davies, J., and Davies, D. (2010). Origins and evolution of antibiotic resistance. Microbiol. Mol. Biol. Rev. 74, 417-433. doi: 10.1128/mmbr.00016-10

D'Costa, V. M., McGrann, K. M., Hughes, D. W., and Wright, G. D. (2006). Sampling the antibiotic resistome. Science 311, 374-377. doi: 10.1126/science. 1120800

Deng, M., Zhu, M.-H., Li, J.-J., Bi, S., Sheng, Z.-K., Hu, F.-S., et al. (2014). Molecular epidemiology and mechanisms of tigecycline resistance in clinical isolates of Acinetobacter baumannii from a Chinese University Hospital. Antimicrob. Agents Chemother. 58, 297-303. doi: 10.1128/AAC.01727-13

Drawz, S. M., and Bonomo, R. A. (2010). Three decades of $\beta$-lactamase inhibitors. Clin. Microbiol. Rev. 23, 160-201. doi: 10.1128/CMR.00037-09

Duchaud, E., Rochat, T., Habib, C., Barbier, P., Loux, V., Guerin, C., et al. (2018). Genomic diversity and evolution of the fish pathogen Flavobacterium psychrophilum. Front. Microbiol. 9:138. doi: 10.3389/fmicb.2018.00138

Duggar, B. M. (1948). Aureomycin: a product of the continuing search for new antibiotics. Annu. N. Y. Acad. Sci. 51, 17-32. doi: 10.1111/j.1749-6632.1948. tb27262.x

Eppink, M. H. M., Overkamp, K. M., Schreuder, H. A., and van Berkel, W. J. H. (1999). Switch of the coenzyme specificity of $p$-hydroxybenzoate hydroxylase. J. Mol. Biol. 292, 87-96.

Eppink, M. H. M., Schreuder, H. A., and van Berkel, W. J. H. (1998). Interdomain binding of $\mathrm{NADPH}$ in p-hydroxybenzoate hydroxylase as suggested by kinetic, crystallographic and modeling studies of histidine 162 and arginine 269 variants. J. Biol. Chem. 273, 21031-21039. doi: 10.1074/jbc.273.33. 21031

Eswaramoorthy, S., Bonanno, J. B., Burley, S. K., and Swaminathan, S. (2006). Mechanism of action of a flavin-containing monooxygenase. Proc. Natl. Acad. Sci. U.S.A. 103, 9832-9837. doi: 10.1073/pnas.0602398103

Favaudon, V. (1977). Oxidation kinetics of 1,5-dihydroflavin by oxygen in nonaqueous solvent. Eur. J. Biochem. 78, 293-307. doi: 10.1111/j.1432-1033.1977. tb11740.x

Favrot, L., Blanchard, J. S., and Vergnolle, O. (2016). Bacterial GCN5-related $N$-acetyltransferases: from resistance to regulation. Biochemistry 55, 989-1002. doi: 10.1021/acs.biochem.5b01269

Fillgrove, K. L., Pakhomova, S., Newcomer, M. E., and Armstrong, R. N. (2003). Mechanistic diversity of fosfomycin resistance in pathogenic microorganisms. J. Am. Chem. Soc. 125, 15730-15731. doi: 10.1021/ja039307z

Finlay, A. C., Hobby, G. L., Pan, S. Y., Regna, P. P., Routien, J. B., Seeley, G. M., et al. (1950). Terramycin, a new antibiotic. Science 111:85. doi: 10.1126/science. 111.2874.85

Fisher, J. F., Meroueh, S. O., and Mobashery, S. (2005). Bacterial resistance to $\beta$-lactam antibiotics: compelling opportunism, compelling opportunity. Chem. Rev. 105, 395-424. doi: 10.1021/cr030102i

Fong, D. H., Burk, D. L., Blanchet, J., Yan, A. Y., and Berghuis, A. M. (2017). Structural basis for kinase-mediated macrolide antibiotic resistance. Structure 25, 750-761. doi: 10.1016/j.str.2017.03.007

Forsberg, K. J., Patel, S., Wencewicz, T. A., and Dantas, G. (2015). The tetracycline destructases: a novel family of tetracycline-inactivating enzymes. Chem. Biol. 22, 888-897. doi: 10.1016/j.chembiol.2015.05.017

Forsberg, K. J., Reyes, A., Wang, B., Selleck, E. M., Sommer, M. O., and Dantas, G. (2012). The shared antibiotic resistome of soil bacteria and human pathogens. Science 337, 1107-1111. doi: 10.1126/science.1220761

Fuoco, D. (2015). Cytotoxicity induced by tetracyclines via protein photooxidation. Adv. Toxicol. 2015:787129. doi: 10.1155/2015/787129

Gerritis, M. M., de Zoete, M. R., Arents, N. L., Kuipers, E. J., and Kusters, J. G. (2002). 16S rRNA mutation-mediated tetracycline resistance in Heliobacter pylori. Antimicrob. Agents Chemother. 46, 2996-3000. doi: 10.1128/AAC.46.9. 2996-3000.2002

Ghavami, A., Labbe, G., Brem, J., Goodfellow, V. J., Marrone, L., Tanner, C. A., et al. (2015). Assay for drug discovery: synthesis and testing of nitrocefin analogues for use as $\beta$-lactamase substrates. Anal. Biochem. 486, 75-77. doi: 10.1016/j.ab. 2015.06.032

Ghisla, S., and Massey, V. (1989). Mechanisms of flavoprotein-catalyzed reactions. Eur. J. Biochem. 181, 1-17. doi: 10.1111/j.1432-1033.1989.tb1 4688.x

Ghosh, S., LaPara, T. M., and Sadowsky, M. J. (2014). Draft genome sequence of Sphingobacterium sp. strain PM2-P1-29, a tetracycline-degrading TetXexpressing aerobic bacterium isolated from agricultural soil. Genome Announc. 2:e00963-14. doi: 10.1128/genomeA.00963-14

Ghosh, S., LaPara, T. M., and Sadowsky, M. J. (2015). Transformation of tetracycline by TetX and its subsequent degradation in a heterologous host. FEMS Microbiol. Ecol. 91:fiv059. doi: 10.1093/femsec/fiv059

Ghosh, S., Sadowsky, M. J., Roberts, M. C., Gralnick, J. A., and LaPara, T. M. (2009). Sphingobacterium sp. strain PM2-P1-29 harbours a functional tet $(X)$ gene encoding for the degradation of tetracycline. J. Appl. Microbiol. 106, 1336-1342. doi: 10.1111/j.1365-2672.2008.04101.x

Gibson, M., Nur-e-alam, M., Lipata, F., Oliveira, M. A., and Rohr, J. (2005). Characterization of kinetics and products of the Baeyer-Villiger oxygenase MtmOIV, the key enzyme of the biosynthetic pathway toward the natural product anticancer drug mithramycin from Streptomyces argillaceus. J. Am. Chem. Soc. 127, 17594-17595. doi: 10.1021/ja055750t

Goldman, P. J., Ryan, K. S., Hamill, M. J., Howard-Jones, A. R., Walsh, C. T., Elliott, S. J., et al. (2012). An unusual role for a mobile flavin in StaC-like indolocarbazole biosynthetic enzymes. Chem. Biol. 19, 855-865. doi: 10.1016/ j.chembiol.2012.05.016

Grossman, T. H., Fyfe, C., O’Brien, W., Hackel, M., Minyard, M. B., Waites, K. B., et al. (2017). Fluorocycline TP-271 is potent against complicated communityacquired bacterial pneumonia pathogens. mSphere 2:e00004-17. doi: 10.1128/ mSphere.00004-17

Grossman, T. H., Starosta, A. L., Fyfe, C., O’Brien, W., Rothstein, D. M., Mikolajka, A., et al. (2012). Target- and resistance-based mechanistic studies with TP-434, a novel fluorocycline antibiotic. Antimicrob. Agents Chemother. 56, 2559-2564. doi: 10.1128/aac.06187-11

Guillaume, G., Ledent, V., Moens, W., and Collard, J. M. (2004). Phylogeny of efflux-mediated tetracycline resistance genes and related proteins revisited. Microb. Drug Resist. 10, 11-26. doi: 10.1089/107662904323047754

Guiney, D. G. Jr., Hasegawa, P., and Davis, C. E. (1984). Expression in Escherichia coli of cryptic tetracycline resistance genes from Bacteroides R plasmids. Plasmid 11, 248-252. doi: 10.1016/0147-619X(84)90031-3

Halling-Sorensen, B., Sengelov, G., and Tjornelund, J. (2002). Toxicity of tetracyclines and tetracycline degradation products to environmentally relevant bacteria, including selected tetracycline-resistant bacteria. Arch. Environ. Contam. Toxicol. 42, 263-271. doi: 10.1007/s00244-001-0017-2

Hinrichs, W., Kisker, C., Duvel, M., Muller, A., Tovar, K., Hillen, W., et al. (1994). Structure of the Tet repressor-tetracycline complex and regulation of antibiotic resistance. Science 264, 418-420. doi: 10.1126/science.8153629

Huijbers, M. M. E., Montersino, S., Westphal, A. H., Tischler, D., and van Berkel, W. J. H. (2014). Flavin dependent monooxygenases. Arch. Biochem. Biophys. 544, 2-17. doi: 10.1016/j.abb.2013.12.005

Izaki, K., and Arima, K. (1963). Disappearance of oxytetracycline accumulation in the cells of multiple drug-resistant Escherichia coli. Nature 200, 384-385. doi: $10.1038 / 200384 \mathrm{a} 0$

Izard, T., and Ellis, J. (2000). The crystal structures of chloramphenicol phosphotransferase reveal a novel inactivation mechanism. EMBO J. 19, 2690-2700. doi: 10.1093/emboj/19.11.2690

Jenner, L., Starosta, A. L., Terry, D. S., Mikolajka, A., Filonava, L., Yusupov, M., et al. (2013). Structural basis for potent inhibitory activity of the antibiotic tigecycline during protein synthesis. Proc. Natl. Acad. Sci. U.S.A. 110, 3812-3816. doi: $10.1073 /$ pnas. 1216691110

Jin, L., Amaya-Mazo, X., Apel, M. E., Sankisa, S. S., Johnson, E., Zbyszynska, M. A., et al. (2007). $\mathrm{Ca}^{2+}$ and $\mathrm{Mg}^{2+}$ bind tetracycline with distinct stoichiometries and linked deprotonation. Biophys. Chem. 128, 185-196. doi: 10.1016/j.bpc.2007. 04.005 
Justice, S. S., Hunstad, D. A., Cegelski, L., and Hultgren, S. J. (2008). Morphological plasticity as a bacterial survival strategy. Nat. Rev. Microbiol. 6, 162-168. doi: $10.1038 /$ nrmicro1820

Kaneko, M., Yamaguchi, A., and Sawai, T. (1985). Energetics of tetracycline efflux system encoded by Tn10 in Escherichia coli. FEBS Lett. 193, 194-198. doi: 10.1016/0014-5793(85)80149-6

Kemal, C., Chan, T. W., and Bruice, T. C. (1977). Reaction of $3 \mathrm{O} 2$ with dihydroflavins. 1. N3,5-dimethyl-1,5-dihydrolumiflavin and 1,5dihydroisoalloxazines. . J. Am. Chem. Soc. 99, 7272-7286. doi: 10.1021/ja0046 $4 \mathrm{a} 030$

Kim, S.-R., Nonaka, L., Oh, M. J., Lavilla-Pitogo, C. R., and Suzuki, S. (2003). Distribution of an oxytetracycline resistance determinant tet(34) among marine bacterial isolates of a Vibrio species. Microbes Environ. 18, 74-81. doi: 10.1264/ jsme2.18.74

King, E. Q., Lewis, C. N., Welch, H., Clark, E. A. Jr., Johnson, J. B., Lyons, J. B., et al. (1950). Clinical observations on the use of Terramycin hydrochloride. J. Am. Med. Assoc. 143, 1-4. doi: 10.1001/jama.1950.02910360003001

Korczynska, M., Mukhtar, T. A., Wright, G. D., and Berghuis, A. M. (2007). Structural basis for streptogramin B resistance in Staphylococcus aureus by virginiamycin B lyase. Proc. Natl. Acad. Sci. U.S.A. 104, 10388-10393. doi: $10.1073 /$ pnas.0701809104

Koteva, K., Cox, G., Kelso, J. K., Surette, M. D., Zubyk, H. L., Ejim, L., et al. (2018). Rox, a rifamycin resistance Enzyme with an unprecedented mechanism of action. Cell Chem. Biol. 25, 403.e5-412.e5. doi: 10.1016/j.chembiol.2018.01.009

Krueger, S. K., and Williams, D. E. (2005). Mammalian flavin-containing monooxygenases: structure/function, genetic polymorphisms and role in drug metabolism. Pharmacol. Ther. 106, 357-387. doi: 10.1016/j.pharmthera.2005. 01.001

Leski, T. A., Bangura, U., Jimmy, D. H., Ansumana, R., Lizewski, S. E., Stenger, D. A., et al. (2013). Multidrug-resistant tet(X)-containing hospital isolates in Sierra Leone. Int. J. Antimicrob. Agents 42, 83-86. doi: 10.1016/j.ijantimicag. 2013.04.014

Leslie, A. G. W. (1990). Refined crystal structure of type III chloramphenicol acetyltransferase at $1.75 \AA$ resolution. J. Mol. Biol. 213, 167-186. doi: 10.1016/ S0022-2836(05)80129-9

Levy, S. B., and McMurry, L. (1978). Plasmid-determined tetracycline resistance involves new transport systems for tetracycline. Nature 276, 90-92. doi: 10. 1038/276090a0

Li, B., Forseth, R. R., Bowers, A. A., Schroeder, F. C., and Walsh, C. T. (2012). A backup plan for self-protection: S-methylation of holomycin biosynthetic intermediates in Streptomyces clavuligerus. Chembiochem 13, 2521-2526. doi: 10.1002/cbic.201200536

Li, Y.-X., Zhong, Z., Hou, P., Zhang, W.-P., and Qian, P.-Y. (2018). Resistance to nonribosomal peptide antibiotics mediated by D-stereospecific peptidases. Nat. Chem. Biol. 14, 381-387. doi: 10.1038/s41589-018-0009-4

Linkevicius, M., Sandegren, L., and Andersson, D. I. (2016). Potential of tetracycline resistance proteins to evolve tigecycline resistance. Antimicrob. Agents Chemother. 60, 789-796. doi: 10.1128/aac.02465-15

Liu, F., and Myers, A. G. (2016). Development of a platform for the discovery and practical synthesis of new tetracycline antibiotics. Curr. Opin. Chem. Biol. 32, 48-57. doi: 10.1016/j.cbpa.2016.03.011

Liu, L.-K., Abdelwahab, H., Martin Del Campo, J. S., Mehra-Chaudhary, R., Sobrado, P., and Tanner, J. J. (2016). The structure of the antibiotic deactivating, $N$-hydroxylating rifampicin monooxygenase. J. Biol. Chem. 291, 21553-21562. doi: 10.1074/jbc.M116.745315

Liu, L.-K., Dai, Y., Abdelwahab, H., Sobrado, P., and Tanner, J. J. (2018). Structural evidence for rifampicin monooxygenase inactivating rifampicin by cleaving its ansa-bridge. Biochemistry 57, 2065-2068. doi: 10.1021/acs.biochem. 8 b00190

Llarrull, L. I., Toth, M., Champion, M. M., and Mobashery, S. (2011). Activation of BlaR1 protein of methicillin-resistant Staphylococcus aureus, its proteolytic processing, and recovery from induction of resistance. J. Biol. Chem. 286, 38148-38158. doi: 10.1074/jbc.M111.288985

Lupien, A., Gingras, H., Leprohon, P., and Ouellette, M. (2015). Induced tigecycline resistance in Streptococcus pneumoniae mutants reveals mutations in ribosomal proteins and rRNA. J. Antimcrob. Chemother. 70, 2973-2980. doi: 10.1093/jac/ dkv211
Mack, S., Xu, Y., and Nodwell, J. R. (2014). The expression of antibiotic resistance genes in antibiotic-producing bacteria. Mol. Microbiol. 93, 391-402. doi: 10. $1111 / \mathrm{mmi} .12689$

Mascotti, M. L., Ayub, M. J., Furnham, N., Thornton, J. M., and Laskowski, R. A. (2016). Chopping and changing: the evolution of the flavin-dependent monooxygenases. J. Mol. Biol. 428, 3131-3146. doi: 10.1016/j.jmb.2016. 07.003

Massey, V. (1994). Activation of molecular oxygen by flavins and flavoproteins. J. Biol. Chem. 269, 22459-22462.

Matthews, B. G., and Guiney, D. G. (1986). Characterization and mapping of regions encoding clindamycin resistance, tetracycline resistance, and a replication function on the Bacteroides R plasmid pCP1. J. Bacteriol. 167, 517-521. doi: 10.1128/jb.167.2.517-521.1986

McCormick, J. R. D., Jensen, E. R., Miller, P. A., and Doerschuk, A. P. (1960). The 6-deoxytetracyclines. Further studies on the relationship between structure and antibacterial activity in the tetracycline series. J. Am. Chem. Soc. 82, 3381-3386. doi: 10.1021/ja01498a037

McPherson, C. J., Aschenbrenner, L. M., Lacey, B. M., Fahnoe, K. C., Lemmon, M. M., Finegan, S. M., et al. (2012). Clinically relevant gram-negative resistance mechanisms have no effect on the efficacy of MC-1, a novel siderophoreconjugated monocarbam. Antimicrob. Agents Chemother. 56, 6334-6342. doi: 10.1128/aac.01345-12

Ming, D.-S., Chen, Q.-Q., and Chen, X.-T. (2017). Analysis of resistance genes in pan-resistant Myroides odoratimimus clinical strain PR63039 using whole genome sequencing. Microb. Pathog. 112, 164-170. doi: 10.1016/j.micpath.2017. 09.012

Montersino, S., and Berkel, W. J. H. V. (2013). “The flavin monooxygenases," in Handbook of Flavoproteins Vol II: Complex Flavoproteins, Dehydrogenases and Physical Methods, eds R. Hille, S. Miller, and B. Palfey (Berlin: De Gruyter), 51-72.

Moore, D. E., Fallon, M. P., and Burt, C. D. (1983). Photo-oxidation of tetracycline-a differential pulse polarographic study. Int. J. Pharm. 14, 133-142. doi: 10.1016/0378-5173(83)90088-1

Moore, I. F., Hughes, D. W., and Wright, G. D. (2005). Tigecycline is modified by the flavin-dependent monooxygenase TetX. Biochemistry 44, 11829-11835. doi: 10.1021/bi0506066

Morar, M., Bhullar, K., Hughes, D. W., Junop, M., and Wright, G. D. (2009). Structure and mechanism of the lincosamide antibiotic adenylyltransferase LinB. Structure 17, 1649-1659. doi: 10.1016/j.str.2009.10.013

Morar, M., Pengelly, K., Koteva, K., and Wright, G. D. (2012). Mechanism and diversity of the erythromycin esterase family of enzymes. Biochemistry 51, 1740-1751. doi: 10.1021/bi201790u

Nelson, M. L., and Levy, S. B. (2011). The history of the tetracyclines. Annu. N. Y. Acad. Sci. 1241, 17-32. doi: 10.1111/j.1749-6632.2011.06354.x

Nguyen, F., Starosta, A. L., Arenz, S., Sohmen, D., Donhofer, A., and Wilson, D. N. (2014). Tetracycline antibiotics and resistance mechanisms. Biol. Chem. 395, 559-575. doi: 10.1515/hsz-2013-0292

Nguyen, V., Nguyen, V., Li, C., and Zhou, G. (2015). The degradation of oxytetracycline during thermal treatments of chicken and pig meat and the toxic effects of degradation products of oxytetracycline on rats. J. Food Sci. Technol. 52, 2842-2850. doi: 10.1007/s13197-014-1306-x

Nonaka, L., Connell, S. R., and Taylor, D. E. (2005). 16S rRNA mutations that confer tetracycline resistance in Helicobacter pylori decrease drug binding in Escherichia coli ribosomes. J. Bacteriol. 187, 3708-3712. doi: 10.1128/JB.187.11. 3708-3712.2005

Nonaka, L., and Suzuki, S. (2002). New $\mathrm{Mg}^{2+}$-dependent oxytetracycline resistance determinant tet34 in Vibrio isolates from marine fish intestinal contents. Antimicrob. Agents Chemother. 46, 1550-1552. doi: 10.1128/AAC.46.5.15501552.2002

O'Callaghan, C. H., Morris, A., Kirby, S. M., and Shingler, A. H. (1972). Novel method for detection of beta-lactamases by using a chromogenic cephalosporin substrate. Antimicrob. Agents Chemother. 1, 283-288. doi: 10.1128/AAC.1.4.283

Ohashi, Y., and Fujisawa, T. (2017). Detection of antibiotic resistance genes in the feces of young adult Japanese. Biosci. Microbiota Food Health 36, 151-154. doi: 10.12938/bmfh.17-004

Olesky, M., Zhao, S., Rosenberg, R. L., and Nicholas, R. A. (2006). Porin-mediated antibiotic resistance in Neisseria gonorrhoeae: Ion, solute, and antibiotic 
permeation through PIB proteins with penB mutations. J. Bacteriol. 188, 2300-2308.

Oliva, B., Gordon, G., McNicholas, P., Ellestad, G., and Chopra, I. (1992). Evidence that tetracycline analogs whose primary target is not the bacterial ribosome cause lysis of Escherichia coli. Antimicrob. Agents Chemother. 36, 913-919. doi: 10.1128/AAC.36.5.913

Palfey, B. A., and McDonald, C. A. (2010). Control of catalysis in flavin-dependent monooxygenases. Arch. Biochem. Biophys. 493, 26-36. doi: 10.1016/j.abb.2009. 11.028

Palmer, A. C., Angelino, E., and Kishony, R. (2010). Chemical decay of an antibiotic inverts selection for resistance. Nat. Chem. Biol. 6, 105-107. doi: 10.1038/ nchembio. 289

Park, B. H., Hendricks, M., Malamy, M. H., Tally, F. P., and Levy, S. B. (1987). Cryptic tetracycline resistance determinant (class F) from Bacteroides fragilis mediates resistance in Escherichia coli by actively reducing tetracycline accumulation. Antimicrob. Agents Chemother. 31, 1739-1743. doi: 10.1128/aac. 31.11.1739

Park, B. H., and Levy, S. B. (1988). The cryptic tetracycline resistance determinant on Tn4400 mediates tetracycline degradation as well as tetracycline efflux. Antimicrob. Agents Chemother. 32, 1797-1800. doi: 10.1128/aac.32.12.1797

Park, J., Gasparrini, A. J., Reck, M. R., Symister, C. T., Elliott, J. L., Vogel, J. P., et al. (2017). Plasticity, dynamics, and inhibition of emerging tetracycline resistance enzymes. Nat. Chem. Biol. 13, 730-736. doi: 10.1038/nchembio.2376

Pawlowski, A. C., Stogios, P. J., Koteva, K., Skarina, T., Evdokimova, E., Savchenko, A., et al. (2018). The evolution of substrate discrimination in macrolide antibiotic resistance enzymes. Nat. Commun. 9:112. doi: 10.1038/ s41467-017-02680-0

Pickens, L. B., and Tang, Y. (2010). Oxytetracycline biosynthesis. J. Biol. Chem. 285, 27509-27515. doi: 10.1074/jbc.R110.130419

Piddock, L. J. V. (2006). Clinically relevant chromosomally encoded multidrug resistance efflux pumps in bacteria. Clin. Microbiol. Rev. 19, 382-402. doi: 10.1128/CMR.19.2.382-402.2006

Ramirez, M. S., and Tolmasky, M. E. (2010). Aminoglycoside modifying enzymes. Drug Resist. Updat. 13, 151-171. doi: 10.1016/j.drup.2010.08.003

Rasmussen, B., Noller, H. F., Daubresse, G., Oliva, B., Misulovin, Z., Rothstein, D. M., et al. (1991). Molecular basis of tetracycline action: identification of analogs whose primary target is not the bacterial ribosome. Antimicrob. Agents Chemother. 35, 2306-2311. doi: 10.1128/AAC.35.11.2306

Reading, C., and Cole, M. (1977). Clavulanic acid: a beta-lactamase-inhibiting beta-lactam from Streptomyces clavuligerus. Antimicrob. Agents Chemother. 11, 852-857. doi: 10.1128/AAC.11.5.852

Rife, C. L., Pharris, R. E., Newcomer, M. E., and Armstrong, R. N. (2002). Crystal structure of a genomically encoded fosfomycin resistance protein (FosA) at 1.19 $\AA$ resolution by MAD phasing off the L-III edge of $\mathrm{Tl}^{+}$. J. Am. Chem. Soc. 124, 11001-11003.

Roberts, M. C. (1996). Tetracycline resistance determinants: mechanisms of action, regulation of expression, genetic mobility, and distribution. FEMS Microbiol. Rev. 19, 1-24. doi: 10.1111/j.1574-6976.1996.tb00251.x

Romero, E., Gómez Castellanos, J. R., Gadda, G., Fraaije, M. W., and Mattevi, A. (2018). Same substrate, many reactions: oxygen activation in flavoenzymes. Chem. Rev. 118, 1742-1769. doi: 10.1021/acs.chemrev.7b00650

Ronn, M., Zhijian, Z., Hogan, P. C., Zhang, W.-Y., Niu, J., Katz, C. E., et al. (2013). Process R\&D of eravacycline: the first fully synthetic fluorocycline in clinical development. Org. Process Res. Dev. 17, 838-845. doi: 10.1021/op4000219

Ross, J. I., Eady, E. A., Cove, J. H., and Cunliffe, W. J. (1998). 16 S rRNA mutation associated with tetracycline resistance in a gram-positive bacterium. Antimicrob. Agents Chemother. 42, 1702-1705.

Ryan, K. S., Chakraborty, S., Howard-Jones, A. R., Walsh, C. T., Ballou, D. P., and Drennan, C. L. (2008). The FAD cofactor of RebC shifts to an IN conformation upon flavin reduction. Biochemistry 47, 13506-13513. doi: 10.1021/bi8 $01229 \mathrm{w}$

Ryerson, C. C., Ballou, D. P., and Walsh, C. (1982). Mechanistic studies on cyclohexanone oxygenase. Biochemistry 21, 2644-2655. doi: 10.1021/ bi00540a011

Santajit, S., and Indrawattana, N. (2016). Mechanisms of antimicrobial resistance in ESKAPE pathogens. BioMed Res. Int. 2016:2475067. doi: 10.1155/2016/247 5067
Schiott, C. R., and Stenderup, A. (1954). Terramycin-, aureomycin- and chloromycetin-dependent bacteria isolated from patients. Acta Pathol. Microbiol. Scand. 34, 410-416. doi: 10.1111/j.1699-0463.1954.tb00838.x

Schnappinger, D., and Hillen, W. (1996). Tetracyclines: antibiotic action, uptake, and resistance mechanisms. Arch. Microbiol. 165, 359-369.

Schwab, J. M., Li, W., and Thomas, L. P. (1983). Cyclohexanone oxygenase: stereochemistry, enantioselectivity, and regioselectivity of an enzyme-catalyzed Baeyer-Villiger reaction. J. Am. Chem. Soc. 105, 4800-4808. doi: 10.1021/ ja00352a044

Spanogiannopoulos, P., Thaker, M., Koteva, K., Waglechner, N., and Wright, G. D. (2012). Characterization of a rifampin-inactivating glycosyltransferase from a screen of environmental Actinomycetes. Antimicrob. Agents Chemother. 56, 5061-5069. doi: 10.1128/AAC.01166- 12

Speer, B. S., Bedzyk, L., and Salyers, A. A. (1991). Evidence that a novel tetracycline resistance gene found on two Bacteroides transposons encodes an NADPrequiring oxidoreductase. J. Bacteriol. 173, 176-183. doi: 10.1128/jb.173.1.176183.1991

Speer, B. S., and Salyers, A. A. (1988). Characterization of a novel tetracycline resistance that functions only in aerobically grown Escherichia coli. J. Bacteriol. 170, 1423-1429. doi: 10.1128/jb.170.4.1423-1429.1988

Speer, B. S., and Salyers, A. A. (1989). Novel aerobic tetracycline resistance gene that chemically modifies tetracycline. J. Bacteriol. 171, 148-153. doi: 10.1128/jb. 171.1.148-153.1989

Stepanek, J. J., Lukezic, T., Teichert, I., Petkovic, H., and Bandow, J. E. (2016). Dual mechanism of action of the atypical tetracycline chelocardin. Biochim. Biophys. Acta 1864, 645-654. doi: 10.1016/j.bbapap.2016.03.004

Stephens, C. R., Conover, L. H., Hochstein, F. A., Regna, P. P., Pilgrim, F. J., Bruning, K. J., et al. (1952). Terramycin. VIII. Structure of aureomycin and terramycin. J. Am. Chem. Soc. 74, 4976-4977. doi: 10.1021/ja0113 9 a533

Stephens, C. R., Murai, K., Brunings, K. J., and Woodward, R. B. (1956). Acidity constants of the tetracycline antibiotics. J. Am. Chem. Soc. 78, 4155-4158. doi: $10.1021 / \mathrm{ja} 01597 \mathrm{a} 081$

Stogios, P. J., Cox, G., Spanogiannopoulos, P., Pillon, M. C., Waglechner, N., Skarina, T., et al. (2016). Rifampin phosphotransferase is an unusual antibiotic resistance kinase. Nat. Commun. 7:11343. doi: 10.1038/ncomms11343

Stone, L. K., Baym, M., Lieberman, T. D., Chait, R., Clardy, J., and Kishony, R. (2016). Compounds that select against the tetracycline-resistance efflux pump. Nat. Chem. Biol. 12, 902-904. doi: 10.1038/nchembio.2176

Suemori, A. (2013). Conserved and non-conserved residues and their role in the structure and function of p-hydroxybenzoate hydroxylase. Protein Eng. Des. Sel. 26, 479-488. doi: 10.1093/protein/gzt026

Sugantino, M., and Roderick, S. L. (2002). Crystal structure of Vat(D): an acetyltransferase that inactivates streptogramin group A antibiotics. Biochemistry 41, 2209-2216. doi: 10.1021/bi011991b

Sun, C., and Xiao, X.-Y. (2017). "Fully synthetic tetracyclines: increasing chemical diversity to combat multidrug-resistant bacterial infections," in Topics in Medicinal Chemistry, ed. J. F. Fisher (Berlin: Springer).

Surette, M. D., and Wright, G. D. (2017). Lessons from the environmental antibiotic resistome. Annu. Rev. Microbiol. 71, 309-329. doi: 10.1146/annurevmicro-090816-093420

Sutcliffe, J. A., O’Brien, W., Fyfe, C., and Grossman, T. H. (2013). Antibacterial activity of eravacycline (TP-434), a novel fluorocycline, against hospital and community pathogens. Antimicrob. Agents Chemother. 57, 5548-5558. doi: 10. 1128/aac.01288-13

Syriopoulou, V. P., Harding, A. L., Goldmann, D. A., and Smith, A. L. (1981). In vitro antibacterial activity of fluorinated analogs of chloramphenicol and thiamphenicol. Antimicrob. Agents Chemother. 19, 294-297. doi: 10.1128/AAC. 19.2.294

Tanaka, S. K., Steenbergen, J., and Villano, S. (2016). Discovery, pharmacology, and clinical profile of omadacycline, a novel aminomethylcycline antibiotic. Bioorg. Med. Chem. 24, 6409-6419. doi: 10.1016/j.bmc.2016.07.029

Thaker, M., Spanogiannopoulos, P., and Wright, G. D. (2010). The tetracycline resistome. Cell. Mol. Life Sci. 67, 419-431. doi: 10.1007/s00018-0090172-6

Therien, A. G., Huber, J. L., Wilson, K. E., Beaulieu, P., Caron, A., Claveau, D., et al. (2012). Broadening the spectrum of $\beta$-lactam antibiotics through inhibition 
of signal peptidase type I. Antimicrob. Agents Chemother. 56, 4662-4670. doi: 10.1128/aac.00726-12

Thiara, A. S., and Cundliffe, E. (1995). Analysis of two capreomycin-resistance determinants from Streptomyces capreolus and characterization of the action of their products. Gene 167, 121-126. doi: 10.1016/0378-1119(95)00702-4

Thompson, M. K., Keithly, M. E., Harp, J., Cook, P. D., Jagessar, K. L., Sulikowski, G. A., et al. (2013). Structural and chemical aspects of resistance to the antibiotic fosfomycin conferred by FosB from Bacillus cereus. Biochemistry 52, 7350-7362. doi: 10.1021/bi4009648

Trieber, C. A., and Taylor, D. E. (2002). Mutations in the 16S rRNA genes of Heliobacter pylori mediate resistance to tetracycline. J. Bacteriol. 184, 2131-2140. doi: 10.1128/JB.187.11.3708-3712.2005

Tripathi, P., Shine, E. E., Healy, A. R., Kim, C. S., Herzon, S. B., Bruner, S. D., et al. (2017). ClbS is a cyclopropane hydrolase that confers colibactin resistance. J. Am. Chem. Soc. 139, 17719-17722. doi: 10.1021/jacs.7b09971

van Berkel, W. J., Kamerbeek, N. M., and Fraaije, M. W. (2006). Flavoprotein monooxygenases, a diverse class of oxidative biocatalysts. J. Biotechnol. 124, 670-689. doi: 10.1016/j.jbiotec.2006.03.044

Volkers, G., Damas, J. M., Palm, G. J., Panjikar, S., Soares, C. M., and Hinrichs, W. (2013). Putative dioxygen-binding sites and recognition of tigecycline and minocycline in the tetracycline-degrading monooxygenase TetX. Acta Crystallogr. D 69, 1758-1767. doi: 10.1107/s0907444913013802

Volkers, G., Palm, G. J., Weiss, M. S., Wright, G. D., and Hinrichs, W. (2011). Structural basis for a new tetracycline resistance mechanism relying on the TetX monooxygenase. FEBS Lett. 585, 1061-1066. doi: 10.1016/j.febslet.2011.03.012

Walsh, C. T., and Wencewicz, T. A. (2013). Flavoenzymes: versatile catalysts in biosynthetic pathways. Nat. Prod. Rep. 30, 175-200. doi: 10.1039/c2np20069d

Wang, P., Bashiri, G., Gao, X., Sawaya, M. R., and Tang, Y. (2013). Uncovering the enzymes that catalyze the final steps in oxytetracycline biosynthesis. J. Am. Chem. Soc. 135, 7138-7141. doi: 10.1021/ja403516u

Wang, Q., Wang, P., and Yang, Q. (2018). Occurrence and diversity of antibiotic resistance in untreated hospital wastewater. Sci. Total Environ. 621, 990-999. doi: 10.1016/j.scitotenv.2017.10.128

Wang, S., Gao, X., Gao, Y., Li, Y., Cao, M., Xi, Z., et al. (2017). Tetracycline resistance genes identified from distinct soil environments in China by functional metagenomics. Front. Microbiol. 8:1406. doi: 10.3389/fmicb.2017. 01406

Ward, J. M., and Hodgson, J. E. (1993). The biosynthetic genes for clavulanic acid and cephamycin production occur as a 'super-cluster' in three Streptomyces. FEMS Microbiol. Lett. 110, 239-242. doi: 10.1111/j.1574-6968.1993.tb06326.x

Warrier, T., Kapilashrami, K., Argyrou, A., Ioerger, T. R., Little, D., Murphy, K. C., et al. (2016). N-methylation of a bactericidal compound as a resistance mechanism in Mycobacterium tuberculosis. Proc. Natl. Acad. Sci. U.S.A. 113, E4523-E4530. doi: 10.1073/pnas.1606590113

Wencewicz, T. A., and Walsh, C. T. (2012). Pseudomonas syringae self-protection from tabtoxinine-beta-lactam by ligase TblF and acetylase Ttr. Biochemistry 51, 7712-7725. doi: 10.1021/bi3011384

Whittle, G., Hund, B. D., Shoemaker, N. B., and Salyers, A. A. (2001). Characterization of the 13-kilobase ermF region of the Bacteroides conjugative transposon CTnDOT. Appl. Environ. Microbiol. 67, 3488-3495. doi: 10.1128/ AEM.67.8.3488-3495.2001

Whittle, G., Shoemaker, N. B., and Salyers, A. A. (2002). The role of Bacteroides conjugative transposons in the dissemination of antibiotic resistance genes. Cell. Mol. Life Sci. 59, 2044-2054. doi: 10.1007/s00018020 0004

Wierenga, R. K., de Jong, R. J., Kalkl, K. H., Hol, W. G. J., and Drenth, J. (1979). Crystal structure of p-hydroxybenzoate hydroxylase. J. Mol. Biol. 131, 55-73. doi: 10.1016/0022-2836(79)90301-2

Wilson, D. N. (2009). The A-Z of bacterial translation inhibitors. Crit. Rev. Biochem. Mol. Biol. 44, 393-433. doi: 10.3109/10409230903307311

Wright, G. D. (2005). Bacterial resistance to antibiotics: enzymatic degradation and modification. Adv. Drug Deliv. Rev. 57, 1451-1470. doi: 10.1016/j.addr.2005. 04.002

Wright, G. D. (2007). The antibiotic resistome: the nexus of chemical and genetic diversity. Nat. Rev. Microbiol. 5, 175-186. doi: 10.1038/nrmicro 1614

Xiao, H., Edwards, T. E., and Ferré-D’Amaré, A. R. (2008). Structural basis for specific, high-affinity tetracycline binding by an in vitro evolved aptamer and artificial riboswitch. Chem. Biol. 15, 1125-1137. doi: 10.1016/j.chembiol.2008. 09.004

Yang, W., Moore, I. F., Koteva, K. P., Bareich, D. C., Hughes, D. W., and Wright, G. D. (2004). TetX is a flavin-dependent monooxygenase conferring resistance to tetracycline antibiotics. J. Biol. Chem. 279, 52346-52352. doi: 10.1074/jbc. M409573200

Yim, G., Huimi Wang, H., and Davies, J. (2007). Antibiotics as signalling molecules. Philos. Trans. R. Soc. Lond. B Biol. Sci. 362, 1195-1200. doi: 10.1098/rstb.2007. 2044

Yu, S., Vosbeek, A., Corbella, K., Severson, J., Schesser, J., and Sutton, L. D. (2012). A chromogenic cephalosporin for $\beta$-lactamase inhibitor screening assays. Anal. Biochem. 428, 96-98. doi: 10.1016/j.ab.2012.06.006

Yuen, P. H., and Sokoloski, T. D. (1977). Kinetics of concomitant degradation of tetracycline to epitetracycline, anhydrotetracycline, and epianhydrotetracycline in acid phosphate solution. J. Pharm. Sci. 66, 1648-1650. doi: 10.1002/jps. 2600661143

Zhanel, G. G., Cheung, D., Adam, H., Zelenitsky, S., Golden, A., Schweizer, F., et al. (2016). Review of eravacycline, a novel fluorocycline antibacterial agent. Drugs 76, 567-588. doi: 10.1007/s40265-016-0545-8

Conflict of Interest Statement: The authors have filed a U.S. patent application (application 20170369864) on methods for treating bacterial infections caused by pathogens expressing tetracycline-inactivating enzymes.

Copyright $(2018$ Markley and Wencewicz. This is an open-access article distributed under the terms of the Creative Commons Attribution License (CC BY). The use, distribution or reproduction in other forums is permitted, provided the original author(s) and the copyright owner are credited and that the original publication in this journal is cited, in accordance with accepted academic practice. No use, distribution or reproduction is permitted which does not comply with these terms. 